\title{
PARTIALLY CONFORMAL TRANSFORMATIONS WITH RESPECT TO (m-1)-DIMENSIONAL DISTRIBUTIONS OF $m$-DIMENSIONAL RIEMANNIAN MANIFOLDS
}

\author{
SHÛKICHI TANNO
}

(Received August 23, 1965)

This paper is devoted to the geometry of transformations which have deep relation with an $(m-1)$-dimensional distribution $D$ of $m$-dimensional Riemannian manifold $M$. A diffeomorphism $\varphi$ of $M$ to another $m$-dimensional Riemannian manifold $N$ induces a mapping of the tangent space at any point $x$ of $M$ to that at $\varphi x$ of $N$. Also $\varphi$ induces a mapping $\varphi_{D}$ of the $(m-1)$ dimensional tangent subspace defined by $D$ to that defined by $\varphi D$. If $\varphi_{D}$ is conformal for any point $x$ of $M$, then we call $\phi$ an $(m-1)$-conformal transformation of $M$ to $N$ with respect to the distribution $D$. A conformal transformation in usual sense is of course an $(m-1)$-conformal transformation. An $(m-1)$-homothetic, or $(m-1)$-isometric transformation is naturally defined by its restriction to $D$. We denote by $D^{\perp}$ the orthocomplementary distribution to $D$. If an $(m-1)$-conformal transformation $\varphi$ maps $D^{\perp}$ to $(\varphi D)^{\perp}$, then it is called special and denoted by an $(m-1)^{s}$-conformal transformation. As $D^{\perp}$ does not always admit a globally defined unit vector field $\zeta$ such that $\zeta_{x} \in D_{x}^{\perp}$ at every point $x$ of $M$, we introduce a symbol ${ }^{\varepsilon} \zeta$ (cf. $\$ 1$ ). By this ${ }^{\varepsilon} \zeta$ we can obtain the equation which characterizes an $(m-1)$-conformal transformation. An $(m-1)$-conformal transformation of $M$ onto itself which preserves $D$ is denoted by an $[m-1]$-conformal transformation.

Examples of such transformation appeared already in the theory of almost contact metric structures. As an almost contact Riemannian manifold admits a globally defined unit vector field $\xi$ (see [14], [15] etc.), we can consider the orthogonal distribution to $\xi$. And a $\phi$-preserving transformation of a contact Riemannian manifold is, in fact, an $[m-1]^{s}$-homothety $([8,9],[17 \sim 20]$, etc.). Further, the existence of such transformations on certain contact Riemannian manifold characterizes the structure of the manifold itself $([19,20])$.

A trivial example is as follows: Let $M, N$ be two Riemannian manifolds with metrics $g, h$ respectively and denote by $R$ a real line, then we can define Riemannian metrics on $M \times R, N \times R$ by $g+k, h+k$ respectively, where $k$ is the usual metric on $R$. If $\phi_{0}: M \rightarrow N$ is a conformal transformation and $f: R \rightarrow R$ is an arbitrary diffeomorphism, then $\varphi: M \times R \rightarrow N \times R$ defined by $\varphi(x, t)=\left(\varphi_{0} x, f(t)\right), t \in R$, is an $[m-1]^{s}$-conformal transformation. 
In $\$ 1$, we give the equation of an $(m-1)$-conformal transformation $\varphi$ and the condition of speciality of $(m-1)$-conformal transformation. In $\$ \S 4 \sim 6$, we calculate $\varphi$-image of the Christoffel's symbol, the Riemannian curvature, the Ricci curvature and scalar curvature. Using these we investigate the properties of $\varphi$ under the additional conditions on $\varphi$ or on manifolds. In $\S 7$, we assume that ${ }^{\varepsilon} \zeta$ is parallel along $D$ and $\phi$ is an $(m-1)^{s}$-homothety, and have a relation of the sectional curvatures (Theorem 7.5). In $\$ 9$, we consider the group $\Pi$ of all $[m-1]$-conformal transformations and its subgroups. It is known that the set of all conformal transformations of a Riemannian manifold is a Lie group ([4]). But generally $\Pi$ is not finite dimensional, so we want to find out the conditions on the manifold and a subgroup of $\Pi$ so that the subgroup is a Lie group. And some answers are given in $\$ 15$ (Theorem 15.9, 15.12).

Chapter II contains some studies of infinitesimal $(m-1)$-conformal transformations. The properties of conformal or infinitesimal conformal transformations (or homothetic, or isometric ones) of Riemannian manifolds are studied by many authors ([1], [3], [10], [16], [22], [23], etc.). In $\$ 16$, we consider the case where $M$ is compact and the scalar curvature is constant and obtain analogous results. Other extended investigation will be seen in other papers.

Contents are as follows:

\section{Chapter I}

1. Definition of an $(m-1)$-conformal transformation

2. Commutability of an $(m-1)^{s}$-homothety and parallel translations

3. The case where each trajectory of $\varepsilon$ is a geodesic

4. Transformation of the Christoffel's symbols

5. $(m-1)^{s}$-conformal and projective transformations

6. The Riemannian curvature, Ricci curvature and scalar curvature

7. The sectional curvatures in the case where ${ }^{8} \zeta$ is parallel along $D$

8. $(m-1)$-Einstein spaces

9. The group of $[m-1]$-conformal transformations

10. (m-1)-conformal transformations of complete or compact manifold $M$

11. Supplementary results

\section{Chapter II}

12. Infinitesimal $(m-1)$-conformal and $[m-1]$-conformal transformations

13. Lie derivative of the Christoffel's symbols by an infinitesimal $(m-1)$ conformal transformation and relations with an infinitesimal affine transformation and projective transformation

14. Lie derivative of the Riemannian curvature by an infinitesimal $(m-1)$ conformal transformation 
15. Lie algebras of infinitesimal $[m-1]$-conformal transformations and Lie transformation groups

16. The case where $M$ is compact and the scalar curvature $R$ is constant

\section{Chapter I}

1. Definition of an $(\mathbf{m}-\mathbf{1})$-conformal transformation. Let $M, N$ be two connected $m$-dimensional Riemannian manifolds $(m \geqq 3)$ of class $C^{\infty}$ and $g, h$ two Riemannian metrics of $M, N$ respectively. First we assume that $M$ admits an $(m-1)$-dimensional distribution $D$, and we fix $D$ throughout the paper. Then we also have an orthocomplementary 1-dimensional distribution. Now we consider a diffeomorphism $\varphi: M \rightarrow N$, and denote by $\phi^{*}$ the dual map of $\boldsymbol{\varphi}$.

DEFINITION. If a diffeomorphism $\varphi: M \rightarrow N$ satisfies the following relation

$$
\left(\phi^{*} h\right)_{x}(u, v)=\alpha(x) g_{x}(u, v)
$$

for any point $x \in M$ and vector fields $u, v$ on $M$ such that $u, v \in D$, where $\alpha$ is a differentiable function on $M$, then we call $\phi$ an $(m-1)$-conformal transformation of $M$ to $N$ with respect to $D$. If $\alpha$ is constant, $\phi$ is an $(m-1)$ homothety. Furthermore if $\alpha=1, \phi$ is an (m-1)-isometry.

In order to express an $(m-1)$-conformal transformation by a tensor equation, let $x$ be an arbitrary point of $M$. Then we can find an open neighborhood $U$ of $x$ and a vector field $\zeta_{U}$ on $U$, such that $\zeta_{U}$ is orthocomplementary to $D$ and a unit vector field i.e., $g\left(\zeta_{U}, \zeta_{U}\right)=1$. For some open covering $\{U\}$ of $M$ we can define $\left\{\zeta_{U}\right\}$ corresponding $\zeta_{U}$ to each $U$ in such a way that $\zeta_{U}=\zeta_{V}$ or $-\zeta_{V}$ holds on the intersection $U \cap V$, if it is not empty. $\left\{\zeta_{U}\right\}$ or its subfamily does not always define a vector field on $M$, so we use the symbol ${ }^{\varepsilon} \zeta=\left\{\zeta_{U}\right\}$. We refer frequently this fixed covering $\{U\}$ in the sequel.

On each neighborhood $U$, we define a 1 -form $w_{U}$ by

$$
w_{v}(u)=g\left(\zeta_{v}, u\right)
$$

for any vector field $u$ on $M$. Then we have

$$
w_{U}\left(\zeta_{v}\right)=1
$$

and $w_{U}=0$ is the equation of the distribution $D$. Similarly we use the notation $^{-}{ }^{\varepsilon} w=\left\{w_{U}\right\}$.

We put $E=\phi^{*} h-\alpha g$, clearly $E(u, v)=0$ if ${ }^{\varepsilon} w(u)={ }^{\varepsilon} w(v)=0$. Next 
we put $E\left(^{\varepsilon} \zeta,{ }^{\varepsilon} \zeta\right)=\beta$, then $\beta$ is a globally defined scalar field because it contains two $\varepsilon^{\prime}$ s, and we define ${ }^{\varepsilon} \theta$ by

$$
{ }^{\varepsilon} \theta(u)=E\left(u,{ }^{\varepsilon} \zeta\right)-\beta^{\varepsilon} w(u)
$$

for any vector field $u$ on $M .{ }^{8} \theta$ is determined as follows: Let $x$ be any point of $M$ then we have some open neighborhood $U \in\{U\}$ of $x$ and $\zeta_{U}, w_{U}$ on $U$. These $\zeta_{U}, w_{V}$ determine $\theta_{U}$ of ${ }^{\varepsilon} \theta$ on $U$. From the definition of $E$ and ${ }^{\varepsilon} \theta$, we see that

$$
{ }^{\varepsilon} \theta\left({ }^{\varepsilon} \zeta\right)=0 .
$$

Then we can verify

$$
\varphi^{*} h=\alpha g+{ }^{\varepsilon} w \otimes{ }^{\varepsilon} \theta+{ }^{\varepsilon} \theta \otimes{ }^{\varepsilon} w+\beta^{\varepsilon} w \otimes{ }^{\varepsilon} w,
$$

where $\otimes$ means the tensor product. To see this, it is enough to compare both sides substituting the pairs $\left.{ }^{8} \zeta,{ }^{8} \zeta\right),\left({ }^{\varepsilon} \zeta, u\right)$ and $(u, v)$ where $u$ and $v$ are vector fields which belong to the distribution $D$. Though ${ }^{\varepsilon} \zeta,{ }^{\varepsilon} w$ and ${ }^{\varepsilon} \theta$ are not tensor fields, restricting ourselves to some neighborhood we consider (1.6) as a tensor equation. Of course, for $U, V \in\{U\}$, the expressions $(1.6)_{U}$ in $U$ and $(1.6)_{V}$ in $V$ are equivalent, because $\varepsilon$ 's appear twice in the last three terms. It is evident that the decomposition of $\phi^{*} h$ given by (1.6) is unique in the sense that (1.5) holds. From the definition of $\beta$ it follows that

$$
\alpha+\beta=h\left(\phi^{\varepsilon \zeta}, \varphi^{\varepsilon \zeta}\right) \cdot \varphi
$$

where we have used, and shall use $\varphi$ to denote also the differential of $\phi$.

As for the distribution $\varphi D$ induced by $\varphi$ on $N$, one has ${ }^{\delta} \xi$ and ${ }^{\delta} \eta$ on $N$ similar to ${ }^{\varepsilon} \zeta$ and ${ }^{\varepsilon} w$ on $M$, satisfying

$$
{ }^{\delta} \eta\left({ }^{\delta} \xi\right)=1, \quad{ }^{\delta} \eta(X)=h\left({ }^{\delta} \xi, X\right)
$$

for any vector field $X$ on $N$. We also fix a covering $\left\{{ }^{\prime} V\right\}$ of $N$.

LEMMA 1.1. For any $(m-1)$-conformal transformation $\phi$, we have $\alpha>0$ and $\alpha+\beta>0$. And the following conditions are eqivalent:

(i) ${ }^{\varepsilon} \theta=0$.

(ii) $\varphi^{\varepsilon \xi}={ }^{\varepsilon \delta} \mu^{\delta} \xi$, for some ${ }^{\varepsilon \delta} \mu$.

In the above lemma, ${ }^{\varepsilon \delta} \mu$ is a symbol of $\left\{\mu_{V^{\prime} V}\right\}$, and $\mu_{V^{\prime} V}$ is a differentiable function on $\varphi U \cap^{\prime} V$. Namely for $x \in M$, if $y=\varphi x$, we take some neighborhoods 
$U$ of $x$ and ' $V$ of $y$, then $\phi \zeta_{U}=\mu_{U^{\prime} V} \xi^{\prime}$ on $\phi U \cap^{\prime} V$. In this case by (1.7), we see that $\mu_{U^{\prime} v}^{2}(\phi p)=\alpha(p)+\beta(p)$ for $p \in U \cap \phi^{-1}(V)$.

Proof. Following the above notation, we show (i) $\rightarrow$ (ii). By the definition of $\varphi D$, we have $\phi^{*} \eta_{, V}=\gamma_{, V U} w_{U}$ for some differentiable function $\gamma_{v_{V U}}$ on $U \cap \varphi^{-1}(V)$. In the following we write this relation by

$$
\phi^{* \delta} \eta={ }^{\delta \varepsilon} \gamma^{\varepsilon} w
$$

Let $u$ be any vector field on $M$ such that ${ }^{\varepsilon} w(u)=0$. If ${ }^{\varepsilon} \theta=0$ holds in (1.6), then we have

$$
h\left(\varphi^{\varepsilon} \zeta, \phi u\right)=\left(\phi^{*} h\right)(\varepsilon \xi, u)=0 .
$$

Thus $\phi^{\varepsilon} \zeta$ is orthocomplementary to $\phi D$. This means that $\phi^{\varepsilon} \zeta$ is proportional to ${ }^{\delta} \xi$. And by (1.9), we have $\phi^{\varepsilon} \xi=\left({ }^{\delta \varepsilon} \gamma \cdot \phi^{-1}\right)^{\delta} \xi$. That is, we get

$$
\varphi^{\varepsilon} \xi={ }^{\varepsilon \delta} \mu^{\delta} \xi,
$$

where

$$
{ }^{\varepsilon \delta} \mu={ }^{\delta \varepsilon} \boldsymbol{\gamma} \cdot \phi^{-1}, \quad{ }^{\delta \varepsilon} \gamma^{2}=\alpha+\beta
$$

In the next place we prove (ii) $\rightarrow$ (i). (1.4) means that

$$
\begin{aligned}
{ }^{\varepsilon} \theta(u) & =h\left(\phi u, \phi^{\varepsilon} \zeta\right) \cdot \varphi-\alpha g\left(u,{ }^{\varepsilon} \zeta\right)-\beta^{\varepsilon} w(u) \\
& =h\left(\phi u,{ }^{\varepsilon \delta} \mu^{\delta} \xi\right) \cdot \phi \\
& =0
\end{aligned}
$$

for any vector field $u \in D$. This completes the proof.

PROPOSITION 1.2. Let $\boldsymbol{\phi}$ be an $(m-1)$-conformal transformations of $M$ to $N$, and let ${ }^{\delta s} K_{N},{ }^{\delta \varepsilon} K_{M}$ be angles determined by $\phi^{\varepsilon \xi}$ and ${ }^{\delta} \xi,{ }^{8} \zeta$ and $\phi^{-1 \delta} \xi$ respectively. Then we have

$$
\begin{gathered}
\cos ^{\delta \varepsilon} K_{N}=\frac{{ }^{\delta \varepsilon} \gamma}{\sqrt{\alpha+\beta}} \varphi^{-1}, \\
\cos ^{\delta \varepsilon} K_{M}=\operatorname{sgn}\left({ }^{\delta \varepsilon} \gamma\right)\left(\frac{\alpha}{2 \alpha+\beta-{ }^{\delta \varepsilon} \gamma^{2}}\right)^{\frac{1}{2}} .
\end{gathered}
$$


Proof. In the formula

$$
\cos ^{\delta \varepsilon} K_{N}=\frac{h\left(\phi^{\varepsilon \zeta}, \delta \xi\right)}{\sqrt{h\left(\phi^{\varepsilon \zeta}, \phi^{\varepsilon \zeta}\right)}}
$$

we substitute $\left(\phi^{*} h\right)(\zeta, \zeta)=\alpha+\beta$ and

$$
\begin{aligned}
h\left(\phi^{\varepsilon} \zeta,{ }^{\delta} \xi\right) & ={ }^{\delta} \eta\left(\phi^{\varepsilon} \zeta\right) \\
& =\left(\phi^{* \delta} \eta\right)\left({ }^{\circledR} \zeta\right) \cdot \phi^{-1}={ }^{\delta \varepsilon} \gamma \cdot \phi^{-1},
\end{aligned}
$$

then we get (1.11). Similarly e have the formula

$$
\cos ^{\delta \varepsilon} K=\frac{g\left({ }^{s} \zeta, \varphi^{-1} \delta \xi\right)}{\sqrt{g\left(\phi^{-1} \xi \xi, \varphi^{-1 \delta} \xi\right)}}
$$

First we have

$$
\begin{aligned}
g\left({ }^{(} \boldsymbol{\xi}, \boldsymbol{\phi}^{-1 \delta \xi}\right) & ={ }^{\varepsilon} w\left(\boldsymbol{\phi}^{-1 \delta} \xi\right) \\
& =\left(\boldsymbol{\phi}^{-1 * \varepsilon} w\right)\left({ }^{\delta} \xi\right) \cdot \boldsymbol{\phi}={ }^{\delta} \boldsymbol{\gamma}^{-1} .
\end{aligned}
$$

And in order to estimate $g\left(\phi^{-1 \delta} \xi, \varphi^{-1 \delta} \xi\right)$, we decompose $\phi^{-1 \delta \xi}$ into orthogonal components as follows:

$$
\boldsymbol{\phi}^{-1 \delta} \xi={ }^{\varepsilon} w\left(\phi^{-1 \delta \xi} \xi\right)^{\varepsilon} \zeta+\left(\phi^{-1 \delta} \xi-{ }^{\varepsilon} w\left(\phi^{-1 \delta} \xi\right)^{\varepsilon} \zeta\right) .
$$

Then we have

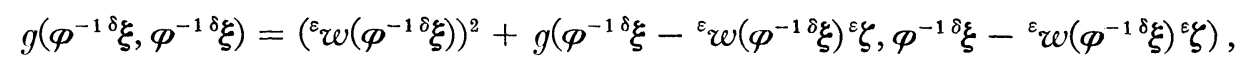

where

$$
\left({ }^{\varepsilon} w\left(\phi^{-1 \delta} \xi\right)\right)^{2}=\left(\left(\phi^{-1 * \varepsilon} w\right)\left({ }^{\delta} \xi\right)\right)^{2}=\left({ }^{\delta \varepsilon} \gamma\right)^{-2},
$$

and

$$
\begin{aligned}
& \alpha g\left(\phi^{-1 \delta} \xi-{ }^{\varepsilon} w\left(\phi^{-1 \delta} \xi\right)^{\varepsilon} \zeta, \phi^{-1 \delta} \xi-{ }^{\varepsilon} w\left(\phi^{-1 \delta} \xi\right) \varepsilon\right) \\
& =h\left({ }^{\delta} \xi-\varphi\left({ }^{\delta \varepsilon} \gamma^{-1} \cdot{ }^{\varepsilon} \zeta\right),{ }^{\delta} \xi-\varphi\left({ }^{\delta \varepsilon} \gamma^{-1} \cdot{ }^{\varepsilon} \zeta\right)\right) \cdot \phi
\end{aligned}
$$

since the 2nd term of the right hand side of (1.15) belongs to the distribution $D$. Then we have 


$$
\begin{aligned}
h\left({ }^{\delta} \xi,{ }^{\delta} \xi\right) & \cdot \boldsymbol{\varphi}-2\left({ }^{\delta \varepsilon} \gamma\right)^{-1} h\left({ }^{\delta} \xi, \varphi^{\varepsilon} \zeta\right) \cdot \boldsymbol{\phi}+\left({ }^{\delta \varepsilon} \gamma\right)^{-2} h\left(\phi^{\varepsilon} \zeta, \phi^{\varepsilon} \zeta\right) \cdot \boldsymbol{\phi} \\
& =1-2\left({ }^{\delta \varepsilon} \gamma\right)^{-1}{ }^{\delta} \eta\left(\phi^{\varepsilon} \zeta\right) \cdot \boldsymbol{\phi}+\left({ }^{\delta \varepsilon} \gamma\right)^{-2}(\alpha+\beta) \\
& =-1+\left({ }^{\delta \varepsilon} \gamma\right)^{-2}(\alpha+\beta) .
\end{aligned}
$$

Therefore, subsituting these into (1.14), we get (1.12).

q.e.d.

It is geometrically obvious that $\phi^{-1}$ is also an $(m-1)$-conformal transformation of $N$ to $M$ with respect to the distribution $\varphi D$, applying $\varphi^{-1 *}$ to (1.6) we have

$$
\begin{gathered}
h=\left(\alpha \cdot \boldsymbol{\phi}^{-1}\right) \boldsymbol{\phi}^{-1 *} g+\boldsymbol{\varphi}^{-1 * \varepsilon} w \otimes \boldsymbol{\phi}^{-1 * \varepsilon} \theta+\boldsymbol{\varphi}^{-1 * \varepsilon} \theta \otimes \boldsymbol{\varphi}^{-1 * \varepsilon} w \\
+\left(\beta \cdot \boldsymbol{\varphi}^{-1}\right) \boldsymbol{\varphi}^{-1 * \varepsilon} w \otimes \boldsymbol{\varphi}^{-1 * \varepsilon} w .
\end{gathered}
$$

Hence

$$
\boldsymbol{\varphi}^{-1 *} g=\left(\frac{1}{\alpha} \cdot \phi^{-1}\right) h+{ }^{\delta} \eta \otimes{ }^{\delta} \lambda+{ }^{\delta} \lambda \otimes{ }^{\delta} \eta+\rho^{\delta} \eta \otimes{ }^{\delta} \eta,
$$

where we have put

$$
\begin{gathered}
{ }^{\delta} \lambda=-\left(\alpha^{-1 \delta \varepsilon} \gamma^{-1} \cdot \phi^{-1}\right)\left(\phi^{-1 * \varepsilon} \theta-{ }^{\varepsilon} \theta\left(\phi^{-1 \delta} \xi\right)^{\delta} \eta\right) \\
\rho=-\left(\alpha^{-1} \beta^{\delta \varepsilon} \gamma^{-2} \cdot \phi^{-1}\right)-2\left(\alpha^{-1 \delta \varepsilon} \gamma^{-1} \cdot \boldsymbol{\varphi}^{-1}\right)^{\varepsilon} \theta\left(\boldsymbol{\phi}^{-1 \delta} \xi\right) \cdot \boldsymbol{\phi}^{-1} .
\end{gathered}
$$

The right hand side of (1.17) contains $\varepsilon$, and so ${ }^{\delta} \lambda$ may be written formally as ${ }^{\varepsilon \delta} \lambda$. However $\varepsilon$ appears twice in each term. Thus ${ }^{\varepsilon \delta} \lambda$ does not depend on the choice of the neighborhood $U \in\{U\}$. Therefore we can omit $\varepsilon$ from ${ }^{\varepsilon \delta} \lambda$. Similarly the right hand side of (1.18) contains $\varepsilon$ and $\delta$ twice in each term respectively. So $\rho$ does not depend on the choice of neighborhoods.

DEFINITION. The most standard $(m-1)$-conformal transformation of $M$ to $N$ is one which satisfies ${ }^{\varepsilon} \theta=0$, we call such an $(m-1)$-conformal transformation a special $(m-1)$-conformal transformation and we denote it by an $(m-1)^{s}$-conformal transformation.

DEFINITION. If we consider an $(m-1)$-conformal transformation $\boldsymbol{\varphi}$ of $M$ onto itself, we sometimes assume that $\varphi$ preserves the distribution. And we denote such $\varphi$ by an [m-1]-conformal transformation. Namely by an $[m-1]$ conformal transformation of $M$ onto $M$ we understand an $(m-1)$-conformal transformation such that $\varphi D=D$. 
ABBreviation. In the subsequent sections, we abbreviate $\varepsilon$ or $\delta$ in ${ }^{\varepsilon} \zeta,{ }^{\delta} \xi,{ }^{\delta} \gamma, \cdots$, frequently in the case where there is no confusion.

2. Commutability of an $(m-1)^{s}$-homothety and the parallel translations. In this section, we study some properties of the $(m-1)^{s}$-homothety of $M$ to $N$ satisfying some additional conditions, concerning the parallel translations with respect to the Riemannian connections. We denote by $\tau$ and $\nabla, ' \tau$ and ' $\nabla$ the parallel translations along certain curves and covariant differentiations with respect to the Riemannian connections for $g, h$ respectively. We utilize the fundamental formula:

$$
\begin{aligned}
2 g\left(\nabla_{X} Z, Y\right)= & X \cdot g(Y, Z)+Z \cdot g(Y, X)-Y \cdot g(Z, X) \\
& +g(X,[Y, Z])+g(Z,[Y, X])-g(Y,[Z, X])
\end{aligned}
$$

for any vector fields $X, Y$ and $Z$.

THEOREM 2.1. Let $\phi$ be an $(m-1)^{s}$-homothety of $M$ to $N$ and suppose that the distribution $D$ in $M$ is completely integrable. If a curve $l=\left\{l_{t}\right.$ : $0 \leqq t \leqq 1\}$ in $M$, joining two points $l_{0}$ and $l_{1}$, is a segment of an integral curve of the distribution $D$. And if $u_{l_{0}}$ is a tangent vector at $l_{0}$ which belongs to $D_{l_{0}}$ and $\tau_{l(t)} u_{l_{0}} \in D_{l_{t}}$ for any $t: 0 \leqq t \leqq 1, l(t)=\left\{l_{s}: 0 \leqq s \leqq t\right\}$, then we have

$$
\varphi_{l_{1}} \tau_{l} u_{l_{0}}={ }^{\prime} \tau_{\varphi l} \varphi_{l_{0}} u_{l_{0}}
$$

PROOF. We can assume that $l$ does not have any self-intersecting point. Let $u$ be a vector field on $M$ such that $u$ coincides with $u_{l_{t}}=\tau_{l(t)} u_{l_{0}}$ on $l_{t}$ : $0 \leqq t \leqq 1$, and belongs to $D$. And let $v$ be a vector field on $M$ such that $v$ is tangential to the curve $l$ and belongs to $D$.

Of course, such $u, v$ exist. In fact, let $\bar{u}$ be any vector field on $M$ which coincides with $u_{l_{t}}$ on $l_{t}$, then $u=\bar{u}-{ }^{\varepsilon} w(\bar{u})^{\varepsilon} \zeta$ satisfies the required property. In this case ${ }^{\varepsilon} w(\bar{u})^{\varepsilon} \zeta$ is a globally defined vector field, since it has two $\varepsilon^{\prime}$ 's.

Now, in (2.1) we set $X=\varphi v, Z=\varphi u$ and replace $g$ by $h$, then we have

$$
\begin{gathered}
2 h\left({ }^{\prime} \nabla_{\varphi v} \phi u, Y\right)=\phi v \cdot h(Y, \phi u)+\phi u \cdot h(Y, \phi v)-Y \cdot h(\phi u, \phi v) \\
+h(\phi v,[Y, \phi u])+h(\phi u,[Y, \phi v])-h(Y,[\phi u, \phi v])
\end{gathered}
$$

for any vector field $Y$ on $N$. By the assumption ${ }^{\varepsilon} \theta=0$ and $w(u)=0$, and by (1.6), we have 


$$
\begin{aligned}
\phi v \cdot h(Y, \phi u) & =v \cdot\left(\phi^{*} h\right)\left(\phi^{-1} Y, u\right) \cdot \phi^{-1} \\
& =v \cdot\left(\alpha g\left(\phi^{-1} Y, u\right)\right) \cdot \phi^{-1} .
\end{aligned}
$$

And we have

$$
\begin{aligned}
h(\boldsymbol{\phi} v,[Y, \boldsymbol{\phi} u]) & =\left(\boldsymbol{\phi}^{*} h\right)\left(v,\left[\boldsymbol{\phi}^{-1} Y, u\right]\right) \cdot \boldsymbol{\phi}^{-1} \\
& =\alpha g\left(v,\left[\boldsymbol{\phi}^{-1} Y, u\right]\right) \cdot \boldsymbol{\varphi}^{-1},
\end{aligned}
$$

$$
h(Y,[\phi u, \phi v])=\alpha g\left(\phi^{-1} Y,[u, v]\right) \cdot \phi^{-1}+\beta w\left(\phi^{-1} Y\right) w([u, v]) \cdot \phi^{-1}
$$

As the distribution $D$ is completely integrable, $w([u, v])=0$ holds good, and so

$$
h\left(\nabla_{\varphi v} \varphi u, Y\right)=\alpha g\left(\nabla_{v} u, \varphi^{-1} Y\right) \cdot \phi^{-1} .
$$

If $u$ is parallel along $l, \nabla_{v} u=0$ holds on $l$ and we have ' $\nabla_{\varphi v} \varphi u=0$ on $\varphi l$. q.e.d.

As a natural consequence, we see that, under the assumption in Theorem 2.1 , if $l$ is not only an integral curve of $D$, but also a geodesic, then $\phi l$ is also geodesic. However this holds without the asumption of the complete integrability of $D$.

THEOREM 2.2. Let $\boldsymbol{\phi}$ be an $(m-1)^{s}$-homothety. If $l$ is an integral curve of $D$ and geodesic with respect to $g$, then $\phi l$ is an integral curve of $\varphi D$ and geodesic with respect to $h$.

PROOF. If $l$ is a geodesic, in the above proof we may assume that $u=v$. In (2.2), we replace $\phi u$ by $\phi v$, then (2.4) replaced $u$ by $v$ holds good, since the 2 nd term of the right hand side of (2.3) is zero. And hence $\nabla_{v} v=0$ on $l$ means ' $\nabla_{\varphi v} \varphi v=0$ on $\varphi l$.

THEOREM 2.3. Suppose that the distribution D is completely integrable and each trajectory of $\varepsilon \xi$ is a geodesic. If an $(m-1)^{s}$-homothetic transformation $\phi$ satisfies $\beta=$ constant. Then, denoting by $l=\left\{l_{t}: 0 \leqq t \leqq 1\right\} a$ segment of the trajectory of ${ }^{\circ} \zeta$, we have

$$
\boldsymbol{\varphi}_{l_{1}} \tau_{l} u_{l_{0}}={ }^{\prime} \tau_{\rho l} \varphi_{l_{0}} u_{l_{0}}
$$

for any tangent vector $u_{l_{0}}$ at $l_{0}$ which belongs to $D_{l_{0}}$.

PROOF. As $\zeta$ is autoparallel and $u_{l_{0}}$ is orthogonal to $\zeta_{l_{0}}, \tau_{l(t)} u_{l_{0}}$ is also 
orthogonal to $\zeta_{l_{t}}$. Let $u$ be a vector field on $M$ such that $u_{l_{\iota}}=\tau_{\text {( }(t)} u_{l_{\mathrm{s}}}$ and $w(u)=0$. Using (2.1) for $\varphi \zeta$ and $\varphi u$, we get

$$
\begin{aligned}
2 h\left({ }^{\prime} \nabla_{\varphi \zeta} \varphi u, Y\right) \cdot \varphi= & 2 \alpha g\left(\nabla_{\zeta} u, \phi^{-1} Y\right)+\beta u \cdot w\left(\phi^{-1} Y\right) \\
& +\beta w\left(\left[\phi^{-1} Y, u\right]\right)-\beta w\left(\phi^{-1} Y\right) w([u, \zeta])
\end{aligned}
$$

for any vector field $Y$ on $N$. If we put $Y=\varphi v$, where $v$ belongs to $D$, as $D$ is integrable, we have

$$
h\left(\nabla_{\phi \zeta} \varphi u, \phi v\right)=\alpha g\left(\nabla_{\xi} u, v\right) \cdot \phi^{-1} .
$$

Next we put $Y=\varphi \zeta$, and notice that

$$
w([\zeta, u])=-L(\zeta) w \cdot u
$$

where $L\left(\zeta_{U}\right)$ means the operator of the Lie derivation with respect to $\zeta_{U}$. It is known that $L\left(\zeta_{U}\right) w_{U}=0$ if and only if each trajectory of $\zeta_{U}$ is a geodesic, since $w(\zeta)=1$. And so we have

$$
\left.h{ }^{\prime} \nabla_{\varphi \zeta} \phi u, \varphi \zeta\right)=\alpha g\left(\nabla_{\zeta} u, \zeta\right) \cdot \varphi^{-1}
$$

By (2.5) and (2.6), $\nabla_{\xi} u=0$ on $l$ means that ${ }^{\prime} \nabla_{{ }_{\zeta}} \varphi u=0$ on $\phi l$.

THEOREM 2.4. Suppose that the distribution $D$ is completely integrable and $l=\left\{l_{t}: 0 \leq t \leq 1\right\}$ is a segment of an integral curve of $D$. If an $(m-1)^{s}$ homothetic transformation $\phi$ satisfies $\beta=$ constant and $\zeta$ is parallel along $l$, then ${ }^{\delta} \xi$ is parallel along $\phi l$.

PROOF. Let $v$ be a vector field stated in the proof of Theorem 2.1. By (2.1) we get

$$
\begin{aligned}
2 h\left({ }^{\prime} \nabla_{\varphi v} \varphi \zeta, Y\right) \cdot \varphi= & 2 \alpha g\left(\nabla_{v} \zeta, \phi^{-1} Y\right)+v \cdot \beta w\left(\phi^{-1} Y\right) \\
& +\beta w\left(\left[\phi^{-1} Y, v\right]\right)-\beta w\left(\phi^{-1} Y\right) w([\zeta, v]) .
\end{aligned}
$$

By the similar argument to the proof of Theorem 2.3 we have

$$
\left.h^{\prime} \nabla_{\varphi v} \varphi \zeta, Y\right)=\alpha g\left(\nabla_{v} \zeta, \phi^{-1} Y\right) \cdot \phi^{-1} .
$$

This completes the proof. 
3. The case where each trajectory of ${ }^{\varepsilon} \zeta$ is a geodesic. In this section we do not necessarily assume that $\alpha$ is constant.

THEOREM 3.1. We assume that $M$ and $N$ admit an $(m-1)^{s}$-conformal transformation $\phi$ such that $\alpha+\beta$ is constant. If each trajectory of ${ }^{\varepsilon}$ is a geodesic, each trajectory of $\delta \xi$ is also geodesic.

PROOF. In (2.1), putting $\varphi \zeta$ and $Y$, we get

$$
\begin{aligned}
2 h\left({ }^{\prime} \nabla_{\phi \zeta} \phi \zeta, Y\right) \cdot \boldsymbol{\phi}= & 2 \zeta \cdot(\alpha+\beta) w\left(\phi^{-1} Y\right)-\phi^{-1} Y \cdot(\alpha+\beta) \\
& +2(\alpha+\beta) w\left(\left[\phi^{-1} Y, \zeta\right]\right) .
\end{aligned}
$$

By the assumption that $\alpha+\beta$ is constant and that each trajectory of $\zeta$ is a geodesic, we see that the right hand side vanishes when we put $Y=\phi \zeta$ and $Y=\phi u$ respectively, $u$ denoting a vector field which belongs to $D$. So we have ${ }^{\prime} \nabla_{\varphi \zeta} \varphi \zeta=0$. As $\varphi \zeta=\mu \xi,|\mu|^{2}=\alpha+\beta$, we see that $\nabla_{\xi} \xi=0$.

THEOREM 3.2. Suppose that each trajectory of ${ }^{\varepsilon} \zeta$ and ${ }^{\circ} \xi$ is a geodesic. Let $\phi$ be an $(m-1)^{s}$-conformal transformation of $M$ to $N$, and $u$ be a vector field on $M$ which belongs to $D$. Then we have $L(u)(\alpha+\beta)=0$.

PrOOF. We utilize (3.1) and putting $Y=\phi u$, we have

$$
\left.2 h{ }^{\prime} \nabla_{\varphi \zeta} \varphi \zeta, \phi u\right) \cdot \varphi=-u \cdot(\alpha+\beta) .
$$

On the other hand, as $(\phi \zeta)_{\phi x}=\mu_{\varphi x} \xi_{\phi x}, \mu_{p x}^{2}=(\alpha+\beta)_{x}, x \in M$, we get

$$
{ }^{\prime} \nabla_{\phi \zeta} \varphi \zeta=\left(\nabla_{\phi \xi} \mu\right) \xi
$$

where we have used ${ }^{\prime} \nabla_{\xi} \xi=0$. By (3.2) and (3.3), we have $u \cdot(\alpha+\beta)=0$.

PROPOSITION 3.3. Suppose that each trajectory of ${ }^{\varepsilon} \zeta$ and ${ }^{\delta} \xi$ is a geodesic. Let $\phi$ be an $(m-1)^{s}$-conformal transformation of $M$ to $N$ such that $\zeta \cdot(\alpha+\beta)$ $=0$. Then $\alpha+\beta$ is constant.

PROOF. Any tangent vector $v_{x}$ at $x \in M$ is written as

$$
v_{x}=\left(v_{x}-w(v) \zeta_{x}\right)+w(v) \zeta_{x}
$$

where $v_{x}-w(v) \zeta_{x} \in D_{x} . \quad$ By Theorem 3.2 we have $\left(v_{x}-w(v) \zeta_{x}\right)(\alpha+\beta)=0$. 
Thus we see that $\alpha+\beta$ is constant.

From Theorem 3.2 we see geometrically the following

PROPOSITION 3.4. Suppose that each trajectory of $\bar{\xi} \xi$ and $\delta \xi$ is a geodesic. Let $\phi$ be an $(m-1)^{s}$-conformal transformation of $M$ to $N$ and let $l$ be a trajectory of $\varepsilon$. If, for each $l$ and for any points $x, y \in l$, we can join $x$ and $y$ by a piecewise differentiable integral curve of $D$. Then $\alpha+\beta$ is constant.

4. Transformation of the Christoffel's symbols. Let $\phi$ be an $(m-1)$ conformal transformation of $M$ to $N$ and $x$ be an arbitrary point of $M$ and $y=\phi x$. On some coordinate neighborhoods $U$ of $x$ and ' $V$ of $y$, we have $w_{U}, \zeta_{U}, \theta_{U}, \xi$ and we write them simply $w, \zeta, \theta, \xi$. We write their components $w^{i}, \xi^{\alpha}$, etc. with respect to the local coordinates $x^{i}, y^{\alpha}: i, \alpha=1,2, \cdots, m$. For convienience, we write $w^{i}$ for $\xi^{i}$ sometimes. Let

$$
G_{i j}=h_{\alpha \beta} \frac{\partial y^{\alpha}}{\partial x^{i}} \frac{\partial y^{\beta}}{\partial x^{j}}
$$

then (1.6) is written as follows:

$$
G_{i j}=\alpha g_{i j}+w_{i} \theta_{j}+\theta_{i} w_{j}+\beta w_{i} w_{j}
$$

We put $\nu=\theta_{i} \theta^{i}-\alpha(\alpha+\beta)$, where $\theta^{i}=g^{i j} \theta_{j}$ and $g^{i j}$ is the inverse matrix of $g_{i j}$. Then the inverse matrix $\left(G^{-1}\right)^{j k}$ of $G_{i j}$ is given by

$$
\left(G^{-1}\right)^{j k}=\frac{1}{\alpha} g^{j k}+\frac{1}{\nu}\left(w^{j} \theta^{k}+\theta^{j} w^{k}\right)-\frac{1}{\alpha \nu} \theta^{j} \theta^{k}+\frac{1}{\nu}\left(\beta-\frac{r}{\alpha}\right) w^{j} w^{k},
$$

where $r=\theta_{j} \theta^{j}$. If $\theta=0,(4.2)$ reduces to

$$
\left(G^{-1}\right)^{j k}=\frac{1}{\alpha} g^{j k}-\frac{\beta}{\alpha(\alpha+\beta)} w^{j} w^{k} .
$$

Denoting by ${ }^{\varphi}\left\{\begin{array}{l}i \\ j k\end{array}\right\},\left\{\begin{array}{l}i \\ j k\end{array}\right\}$ the Christoffel's symbols with respect to $G_{i j}, g_{i j}$ respectively, generally we see that

$$
\nabla_{k}\left(\phi^{*} h\right)_{i j}-\left(\phi^{*}\left({ }^{\prime} h\right)\right)_{k i j}=\left({ }^{\phi}\left\{\begin{array}{l}
r \\
i k
\end{array}\right\}-\left\{\begin{array}{l}
r \\
i k
\end{array}\right\}\right)\left(\phi^{*} h\right)_{r j}+\left({ }^{\phi}\left\{\begin{array}{l}
r \\
j k
\end{array}\right\}-\left\{\begin{array}{l}
r \\
j k
\end{array}\right\}\right)\left(\phi^{*} h\right)_{i r}
$$

holds good, where $\nabla$ and ' $\nabla$ are covariant differentiation with respect to $g$ 
and $h$. The second term of the left hand side of (4.4) vanishes. Making up (4.4) in the simplified form, we get

$$
2\left({ }^{\Phi}\left\{\begin{array}{l}
r \\
j k
\end{array}\right\}-\left\{\begin{array}{l}
r \\
j k
\end{array}\right\}\right) G_{r i}=\nabla_{j} G_{k i}+\nabla_{k} G_{i j}-\nabla_{i} G_{j k} .
$$

We calculate ${ }^{\rho}\left\{\begin{array}{l}i \\ j k\end{array}\right\}$ by (4.1), (4.2) and (4.5) and we have

$$
\begin{aligned}
2^{\Phi}\left\{\begin{array}{l}
i \\
j k
\end{array}\right\}= & 2\left\{\begin{array}{l}
i \\
j k
\end{array}\right\}+\frac{1}{\alpha}\left(\alpha_{j} \delta_{k}^{i}+\alpha_{k} \delta_{j}^{i}-\alpha^{i} g_{j k}\right) \\
& -\frac{1}{\alpha} \beta^{i} w_{j} w_{k}+\frac{2 \beta}{\alpha} w_{(k}\left(w_{, j}^{i}-w_{j)}{ }^{i}\right) \\
& -\frac{w^{i}}{\nu}\left\{\left(\beta-\frac{r}{\alpha}\right)(\zeta \alpha) g_{j k}+\left(\beta-\frac{r}{\alpha}\right)(\zeta \beta) w_{j} w_{k}\right. \\
& -2\left(\beta-\frac{r}{\alpha}\right) \alpha_{(j} w_{k)}+2 \alpha \beta_{(j} w_{k)}+2 \alpha\left(\beta-\frac{r}{\alpha}\right) w_{(j, k)} \\
& \left.+2 \beta\left(\beta-\frac{r}{\alpha}\right) w_{\left(j, w_{k), s} w^{s}\right\}}\right\}+([\theta])
\end{aligned}
$$

where we have used the notations $\nabla_{i} w_{j}=w_{j, i}, \alpha_{i}=\partial \alpha / \partial x^{i}, \alpha^{k}=g^{k i} \alpha_{i}, \beta^{k}$ $=g^{k i} \beta_{i}, \zeta \alpha=w^{i} \alpha_{i}$, and ( ) for indices means half of the sum of two terms interchanged two indices, for example

$$
2 w_{(k} w_{, j}^{i}=w_{k} w_{, j}^{i}+w_{j} w_{, k}^{i},
$$

and finally we have put

$$
\begin{aligned}
([\theta])= & 2 \alpha^{-1} g^{h i}\left\{w_{(k}\left(\theta_{|h|, j)}-\theta_{j), h}\right)+\theta_{(k}\left(w_{|h|, j)}-w_{j), h}\right)\right\} \\
& +\nu^{-1} w^{i}\left\{2 \alpha_{(j} \theta_{k)}-(\theta \alpha) g_{j k}-(\theta \beta) w_{j} w_{k}-2 \alpha \theta_{(j, k)}\right. \\
& -2 \beta w_{(j}\left(w_{k), s}-w_{|s|, k)}\right) \theta^{s} \\
& -2 w_{(j,}\left(\theta_{k), s}-\theta_{|s|, k)}\right) \theta^{s} \\
& -2 \theta_{(j}\left(w_{k), s}-w_{|s|, k)}\right) \theta^{s} \\
& \left.-2\left(\beta-r \alpha^{-1}\right)\left(\theta_{(j} w_{k), s} w^{s}+w_{(j}\left(\theta_{k), s}-\theta_{|s|, k)}\right) w^{s}\right)\right\} \\
& +\nu^{-1} \theta^{i}\left\{\left(\alpha^{-1} \theta \alpha-\zeta \alpha\right) g_{j k}+\left(\alpha^{-1} \theta \beta-\zeta \beta\right) w_{j} w_{k}\right. \\
& +2 \theta_{(j, k)}-2 \alpha w_{(j, k)}+2 \alpha_{(j} w_{k)}+2 \beta_{(j} w_{k)}
\end{aligned}
$$




$$
\begin{aligned}
& -2 \alpha^{-1} \alpha_{(j} \theta_{k)}-2 \beta w_{(j} w_{k), s} w^{s} \\
& -2\left(w_{(j}\left(\theta_{k), s}-\theta_{|s|, k)}\right) w^{s}+\theta_{(j} w_{k), s} w^{s}\right) \\
& +2 \alpha^{-1} \beta w_{(j}\left(w_{k), s}-w_{|s|, k)}\right) \theta^{s} \\
& \left.+2 \alpha^{-1}\left(w_{(j}\left(\theta_{k), s}-\theta_{|s|, k)}\right) \theta^{s}+\theta_{(j}\left(w_{k), s}-w_{|s|, k)}\right) \theta^{s}\right)\right\} .
\end{aligned}
$$

Contracting with respect to $i$ and $k$, we have

(4. 8) $2^{\varphi}\left\{\begin{array}{l}k \\ j k\end{array}\right\}=2\left\{\begin{array}{l}k \\ j k\end{array}\right\}+\left(m \alpha^{-1}+\beta \nu^{-1}-2 r \alpha^{-1} \nu^{-1}\right) \alpha_{j}-\alpha \nu^{-1} \beta_{j}+\nu^{-1} r_{j}$.

THEOREM 4.1. Let $\phi$ be an $(m-1)^{s}$-conformal transformation of $M$ to $N$. If $\phi$ is an affine transformation, we have

(1) $\alpha$ and $\beta$ are constant.

And as a necessary condition that $M$ and $N$ admit such $\phi$ satisfying $\beta \neq 0$, we have

(2) ${ }^{\varepsilon} \zeta$ is a parallel field.

PROOF. By the assumption the last term of the right hand side of (4.7) vanishes and ${ }^{\varphi}\left\{\begin{array}{l}i \\ j k\end{array}\right\}=\left\{\begin{array}{l}i \\ j k\end{array}\right\}$ holds good. Transvecting (4.6) with $w^{j} w_{i}$, we get

$$
\alpha_{k}+\beta_{k}=0
$$

And from (4.8), it follows that

$$
(m(\alpha+\beta)-\beta) \alpha_{k}+\alpha \beta_{k}=0 .
$$

(4.9) and (4.10) give the following relation

$$
(m-1)(\alpha+\beta) \alpha_{k}=0
$$

Thus $\alpha$ is constant and, by (4.9), $\beta$ is also constant.

In the next place we prove (2). Transvecting (4.6) with $w^{j} w^{k}$ and using the fact that $\alpha$ and $\beta$ are constant, we get

$$
\beta w^{i}{ }_{j} w^{j}=0 .
$$

If $\beta \neq 0$, by (4.11) we get $w_{i, j} w^{j}=0$. Transvecting (4.6) with $w_{i}$, we have

$$
\beta\left(w_{j, k}+w_{k, j}\right)=0 .
$$


Thus $\zeta_{U}$ has a property of a Killing vector field. Transvecting (4.6) with $w^{k}$ we have

$$
\beta\left(w_{i, j}-w_{j, i}\right)=0 .
$$

Hence $\zeta$ is a parallel field.

q.e.d.

REMARK. In (2) of the Theorem 4.1 , the assumption $\beta \neq 0$ means that $\phi$ is an essentially $(m-1)^{s}$-conformal transformation.

As a converse, next theorem follows from (4.6) immediately.

THEOREM 4.2. Suppose that $\phi$ is an $(m-1)^{s}$-homothetic transformation of $M$ to $N$ such that $\beta$ is constant. If ${ }^{\xi} \xi$ is a parallel field. Then $\varphi$ is an affine transformation.

5. $(m-1)^{s}$-conformal and projective transformation. By definition a projective transformation $\phi$ of $M$ to $N$ is one which transforms the system of geodesics in $M$ into the same system in $N$. Namely as a necessary and sufficient condition that $\phi$ is a projective transformation we have

$$
2^{\Phi}\left\{\begin{array}{l}
i \\
j k
\end{array}\right\}-2\left\{\begin{array}{l}
i \\
j k
\end{array}\right\}=2 \delta_{j}^{i} \psi_{k}+2 \delta_{k}^{i} \psi_{j}
$$

where $\psi$ is a differentiable function on $M$.

Suppose that $\phi$ is an $(m-1)^{s}$-conformal and at the same time projective transformation. Contracting with respect to $i$ and $j$ in (5.1) and using (4.8), we get

$$
2(m+1) \psi_{k}=\frac{m(\alpha+\beta)-\beta}{\alpha(\alpha+\beta)} \alpha_{k}+\frac{1}{\alpha+\beta} \beta_{k} .
$$

Thus, if $\alpha$ and $\beta$ are constant, $\psi_{k}=0$ holds good and we see that $\phi$ is an affine transformation.

PROPOSITION 5.1. Let $\phi$ be an $(m-1)^{s}$-conformal and at the same time projective transformation. Then we have

$$
2 d \psi=d \log \alpha .
$$

ProOF. Transvecting (4.6) with $w^{j} w_{i}$ and utilizing (5.1), we get 


$$
2 \zeta \psi \cdot w_{k}+2 \psi_{k}=\frac{1}{\alpha+\beta}\left(\alpha_{k}+\beta_{k}\right)
$$

Transvecting (5.2) and (5.4) with $w^{k}$, we obtain

$$
\begin{gathered}
2(m+1) \zeta \psi=\frac{m(\alpha+\beta)-\beta}{\alpha(\alpha+\beta)} \zeta \alpha+\frac{1}{\alpha+\beta} \zeta \beta, \\
4 \zeta \psi=\frac{1}{\alpha+\beta}(\zeta \alpha+\zeta \beta) .
\end{gathered}
$$

Eliminating $\zeta \psi$ from (5.5) and (5.6), we get

$$
(\alpha+2 \beta) \zeta \alpha=\alpha \zeta \beta
$$

By (5.6) and (5.7), we get $2 \zeta \psi=\frac{1}{\alpha} \zeta \alpha$. And so, by virtue of (5.2), (5.4) is written as

$$
((m-1) \beta-\alpha) \alpha_{k}-m \alpha \beta_{k}+(m+1)(\alpha+\beta) \zeta \alpha \cdot w_{k}=0 .
$$

Transvecting (4.6) with $g^{j k} w_{\imath}$ and using (5.1), (5.7) and $2 \alpha \zeta \psi=\zeta \alpha$, we get

$$
(m-1) \zeta \alpha=2 \beta w_{, i}^{i} \text {. }
$$

Transvecting (4.6) with $w^{j} w^{k}$ and $g^{j k}$ respectively and using (5.1), (5.2), (5.7) and (5.9), we have

$$
\begin{aligned}
& 2(\alpha+\beta) \zeta \alpha \cdot w_{k}-\alpha\left(\alpha_{k}+\beta_{k}\right)+2 \alpha \beta w_{k, i} w^{i}=0 \\
& \frac{(m+1) \alpha+2 \beta}{\alpha} \zeta \alpha \cdot w_{k}+\frac{(\alpha+\beta)\left(2-m-m^{2}\right)+2 \beta}{(\alpha+\beta)(m+1)} \alpha_{k} \\
& \quad-\frac{(\alpha+\beta)(m+1)+2 \alpha}{(\alpha+\beta)(m+1)} \beta_{k}+2 \beta w_{k, i} w^{i}=0 .
\end{aligned}
$$

Eliminating $w_{k, i} w^{i}$ from the two above equations

(5.12) $\left(\left(3-m^{2}\right)(\alpha+\beta)+2 \beta\right) \alpha_{k}-2 \alpha \beta_{k}+(m-1)(m+1)(\alpha+\beta) \zeta \alpha \cdot w_{k}=0$.

Eliminating $w_{k}$ from (5.8) and (5.12), as $m>2$, we get

$$
(\alpha+2 \beta) \alpha_{k}-\alpha \beta_{k}=0
$$


From (5.2) and (5.13), we deduce (5.3).

THEOREM 5.2. Let $\phi$ be an $(m-1)^{s}$-homothety and at the same time projective transformation. Then $\phi$ is an affine transformation. Further $\beta$ is constant and " $\mathrm{s}$ is a parallel field.

ProOF. As $\alpha$ is constant, we have $\psi_{k}=0$ by Proposition 5.1, hence $\varphi$ is an affine transformation. Then we can apply Theorem 4.1.

THEOREM 5.3. Let $\phi$ be an $(m-1)^{s}$-conformal transformation and at the same time projective transformation such that $\alpha+\beta$ is constant. Then we see that $\alpha$ and $\beta$ are constant and $\varphi$ is an affine transformation.

PROOF. This is an immediate consequence of (5.13).

COROLLARY 5.4. Let $\phi$ be an $(m-1)^{s}$-conformal transformation of $M$ onto itself and at the same time projective transformation such that $\phi^{8} \xi$ $= \pm \xi$. Then $\phi$ is an affine transformation.

\section{The Riemannian curvature, Ricci curvature and scalar curvature.} Let $\varphi$ be an $(m-1)$-conformal transformation of $M$ to $N$. We denote by $R_{j k l}^{i}$, $R_{j k}, R$ and ${ }^{\varphi} R_{j k l}^{i},{ }^{\varphi} R_{j k},{ }^{\varphi} R$ the Riemannian curvatures, Ricci curvatures, scalar curvatures with respect to $g$ and $\varphi^{*} h=\left(G_{i j}\right)$ respectively. First we have

$$
{ }^{\varphi} R_{j k l}^{i}=R_{j k l}^{i}+W_{j k, l}^{i}-W_{j l, k}^{i}+W_{r l}^{i} W_{j k}^{r}-W_{r k}^{i} W_{j l}^{r},
$$

where covariant derivative $($,$) is the one with respect to \left\{\begin{array}{l}i \\ j k\end{array}\right\}$ and

$$
\left.W_{j k}^{i}=\stackrel{p}{q} \begin{array}{l}
i \\
j k
\end{array}\right\}-\left\{\begin{array}{l}
i \\
j k
\end{array}\right\} .
$$

The verification of (6.1) is as follows: When we calculate ${ }^{\varphi} R_{j k l}^{i}$ by $\left\{\begin{array}{l}i \\ j k\end{array}\right\}+W_{j k}^{i}$, we have the right side of (6.1) and the terms which contain $\left\{\begin{array}{l}i \\ j k\end{array}\right\}$,s. At any point $x$ of $M$, we can find local coordinates $x^{i}$ such that $\left\{\begin{array}{l}i \\ j k\end{array}\right\}_{x}=0$ holds good. Then we have (6.1) at $x$, and as (6.1) is a tensor equation, we get (6.1).

Contracting with respect to $i$ and $l$, we have the relation of the Ricci curvatures by;

$$
{ }^{\phi} R_{j k}=R_{j k}+W_{j k, i}^{i}-W_{j i, k}^{i}+W_{r i}^{i} W_{j k}^{r}-W_{r k}^{i} W_{j i}^{r} .
$$


Transvecting (6.3) with (4.2), we get

(6. 4) ${ }^{\varphi} R=\frac{1}{\alpha} R+\frac{1}{\alpha}\left(W_{j k}^{i} g^{j k}\right), i_{i}-\frac{1}{\alpha}\left(W_{j i}^{i}\right)^{j}+\frac{1}{\alpha} W_{i r}^{i} W_{j k}^{r} g^{j k}-\frac{1}{\alpha} W_{k r}^{i} W_{j i}^{r} g^{j k}$

$$
\begin{gathered}
+\frac{1}{\nu}\left(\beta-\frac{r}{\alpha}\right)\left\{R_{1}(\zeta, \zeta)+W_{j k, i}^{i} \tau \omega^{j} w^{k}-W_{j i, k}^{i} w w^{j} w^{k}\right. \\
\left.+W_{i r}^{i} W_{j k}^{r} w^{j} w^{k}-W_{k r}^{i} W_{j i}^{r} w^{k} w^{j}\right\} \\
+\frac{1}{\nu}\left\{2 R_{1}(\theta, \zeta)+2 W_{j k, i}^{i} \theta^{j} w^{k}-W_{j i, k}^{i}\left(w^{j} \theta^{k}+w^{k} \theta^{j}\right)\right. \\
\left.+2 W_{i r}^{i} W_{j k}^{r} w^{j} \theta^{k}-2 W_{k r}^{i} W_{j i}^{r} \theta^{k} w^{j}\right\} \\
+\frac{1}{\alpha \nu}\left\{-R_{1}(\theta, \theta)+\left(-W_{j k, i}^{i}+W_{j i, k}^{i}-W_{i r}^{i} W_{j k}^{r}+W_{k r}^{i} W_{j i}^{r} \theta^{j} \theta^{k}\right\},\right.
\end{gathered}
$$

where $R_{1}$ denotes the Ricci curvature.

As a special case, we consider an $(m-1)^{s}$-homothetic transformation of $M$ to $N$ assuming that $\zeta$ is a parallel field. By (4.6), we have

$$
2 W_{j k}^{i}=-\frac{1}{\alpha} \beta^{i} w_{j} w_{k}+\frac{w^{i}}{\alpha(\alpha+\beta)}\left(\beta(\zeta \beta) w_{j} w_{k}+2 \alpha \beta_{(j} w_{k)}\right) .
$$

Then by (6.3) we have

$$
\begin{aligned}
& 4^{\varphi} R_{j k}=4 R_{j k}+\frac{2}{\alpha+\beta}\left(\beta_{j, l} w w^{l} w_{k}+\beta_{k, l} w^{l} w_{j}-\beta_{j, k}\right) \\
&+\frac{1}{\alpha(\alpha+\beta)^{2}} w_{j} w_{k}\left\{-2(\alpha+\beta)^{2} \beta^{i},{ }_{i}+2 \beta(\alpha+\beta) \beta_{i, l} w w^{i} w\right. \\
&\left.\quad+(\alpha+\beta) \beta_{r} \beta^{r}-\beta(\zeta \beta)^{2}\right\} \\
&+\frac{1}{(\alpha+\beta)^{2}}\left(\beta_{j} \beta_{k}-2(\zeta \beta) \beta_{(j} w_{k)}\right)
\end{aligned}
$$

And finally from (4.3), we have

$$
4^{\varphi} R=\frac{4}{\alpha} R+\frac{4}{\alpha(\alpha+\beta)}\left(\beta_{j, i} w^{j} w^{l}-\beta_{, i}^{i}\right)+\frac{2}{\alpha(\alpha+\beta)^{2}}\left(\beta_{r} \beta^{r}-(\zeta \beta)^{2}\right),
$$

where we have used $R_{i j} w^{i} w^{j}=0$, which follows from the fact that $w^{i}$ is a parallel field. 
Now, next Proposition is evident:

PROPOSITION 6.1. Suppose that ${ }^{\text {s }} \zeta$ is a parallel field and $\varphi$ is an $(m-1)^{s}$ homothety of $M$ to $N$ such that $\beta$ is constant. Then the scalar curvatures are in the relation ' $R_{\varphi x}=\frac{1}{\alpha} R_{x}, x \in M$. Particularly if both scalar curvatures are constant and equal to $R \neq 0$, then $\phi$ is an $(m-1)^{s}$-isometry.

As usual, we denote by $\delta$ the dual of $d$ (i.e. codifferentiation), then we have $\beta^{i}{ }_{, i}=-\delta d \beta$.

THEOREM 6.2. Suppose that ${ }^{8} \xi$ is a parallel field and $\phi$ is an $(m-1)^{s}$ homothety of $M$ onto itself such that $\delta d \beta=0$ and $\xi \beta=0$ hold good.

(i) If $R=0, \beta$ is constant.

(ii) If $R=$ constant $<0$, then $\alpha \leqq 1$.

(iii) If $R=$ constant $>0$, then $\alpha \geqq 1$.

In (ii) and (iii) equality holds if and only if $\beta$ is constant.

PROOF. If $R$ is constant by (6.7) we get

$$
\frac{1}{(\alpha+\beta)^{2}} \beta_{r} \beta^{r}=2(\alpha-1) R
$$

where we have used $\beta_{j, l} w^{j} w^{l}=\left(\beta_{j} w^{j}\right)_{, l} w^{l}=0$. From (6.8), (i), (ii) and (iii) follow. If $\alpha=1$, then $\beta_{r} \beta^{r}=0$ holds, hence $\beta$ is constant,

THEOREM 6.3. Suppose that ${ }^{\text {}} \boldsymbol{y}$ is a parallel field and $\varphi$ is an $(m-1)^{s}$ homothety of $M$ onto itself such that $\beta$ is constant. If the scalar curvature is bounded and not equal to 0 somewhere, then $\phi$ is an $(m-1)^{s}$-isometry.

Proof. By (6.7) we have ${ }^{\varphi} R=\frac{1}{\alpha} R$ namely $R_{\varphi_{x}}=\frac{1}{\alpha} R_{x}, x$ being a point at which $R_{x}$ is not zero. Then by iteration, we have $R_{\phi^{k} x}=\left(\frac{1}{\alpha}\right)^{k} R_{x}$. As $R$ is bounded, we can conclude that $\alpha=1$.

THEOREM 6.4. Suppose that ${ }^{\text {\& }} \mathrm{\zeta}$ is a parallel field and $\varphi$ is an $(m-1)^{s}$ homothety of $M$ onto itself which preserves the Ricci curvature.

(i) If $\delta d \beta=0$ and $\zeta \beta=0$, then $\beta$ is constant.

(ii) If $M$ is compact orientable, then $d \beta$ is proportional to $w$.

(iii) If $M$ is compact and there exists a point $x$ such that $R_{x} \neq 0$, then $\alpha=1$. 
PROOF. Noticing that ${ }^{\Phi} R_{j k}=R_{j k}$, we transvect (6.6) with $w^{j} w^{k}$, and get

$$
2 \beta_{j, l} w^{j} w^{l}-2 \beta_{, i}^{i}+\frac{1}{\alpha+\beta} \beta_{r} \beta^{r}-\frac{1}{\alpha+\beta}(\zeta \beta)^{2}=0 .
$$

Then (i) is clear. To prove (ii), if we integrate (6.9) over $M$, we have

$$
\int_{M} \frac{1}{\alpha+\beta}\left(\beta_{r} \beta^{r}-(\zeta \beta)^{2}\right) d \sigma=0
$$

where we have used $\int_{M} \beta_{j, l} w^{j} w^{l} d \sigma=\int_{M}\left(\beta_{j} w^{j} w^{l}\right),{ }_{l} d \sigma=0$, and $d \sigma$ denotes the volume element of $M$. As $\alpha+\beta$ is positive and $\left(\beta_{r} \dot{\beta}^{r}-(\zeta \beta)^{2}\right)=\left(\beta_{r}-(\zeta \beta) w_{r}\right)$ $\times\left(\beta^{r}-(\zeta \beta) w^{r}\right)$ is non-negative, we have $\beta_{r}=(\zeta \beta) w_{r}$, namely $d \beta$ is proportional to $w$. On the other hand, transvecting ${ }^{\phi} R_{j k}=R_{j k}$ with $\left(G^{-1}\right)^{j k}$ in (6.6), we have ${ }^{\varphi} R=\frac{1}{\alpha} R$. As $M$ is compact, $R$ is bounded, so we have $\alpha=1$.

REMARK. Assume that $\zeta$ is a parallel field and $\phi$ is an $[m-1]^{s}$-homothety of $M$ onto itself, then we have (i) and (ii). Because ${ }^{\Phi} R_{1}(\zeta, \zeta)=\mu^{2} R_{1}(\zeta, \zeta) \cdot \varphi=0$.

7. The sectional curvatures in the case where ${ }^{\varepsilon} \zeta$ is parallel along $D$. We say that ${ }^{8} \zeta$ is parallel along $D$, if $\nabla_{u} \zeta=0$ holds for any vector field $u$ which belongs to the distribution $D$ i.e. $w(u)=0$. First we prove

LEMMA 7.1. If ${ }^{8} \boldsymbol{y}$ is parallel along $D$, then $D$ is completely integrable.

Proof. Suppose that $u$ and $v$ belong to $D$, then we have

$$
w\left(\nabla_{v} u\right)=\nabla_{v}(w(u))-\nabla_{v} w \cdot u=0,
$$

from which we have

$$
w([u, v])=w\left(\nabla_{u} v-\nabla_{v} u\right)=0 .
$$

This completes the proof.

LEMMA 7.2. If ${ }^{\varepsilon} \zeta$ is parallel along $D$ and if $\phi$ is an $(m-1)^{s}$-homothety of $M$ to $N$. Let $u, v$ be vector fields which belong to $D$, then we have

$$
{ }^{\prime} \nabla_{\varphi v} \varphi u=\varphi \nabla_{v} u \text {. }
$$


PROOF. If $u$ and $v$ belong to $D$ and $\phi$ is an $(m-1)^{s}$-homothety we have (2.4), equivalently

$$
h\left(^{\prime} \nabla_{\varphi v} \varphi u, Y\right)=\alpha\left(\phi^{-1 *} g\right)\left(\phi \nabla_{v} u, Y\right)
$$

for any vector field $Y$ on $N$. By (1.16), we have

$$
\phi^{-1 *} g=\frac{1}{\alpha} h-\left(\frac{\beta}{\alpha(\alpha+\beta)} \cdot \varphi^{-1}\right) \eta \otimes \eta
$$

And we get

$$
(\eta \otimes \eta)\left(\varphi \nabla_{v} u, Y\right)=\eta\left(\varphi \nabla_{v} u\right) \cdot \eta(Y)=\gamma w\left(\nabla_{v} u\right) \varphi^{-1} \cdot \eta(Y)=0 .
$$

Then by virtue of (7.4), (7.5) and (7.6), we get

$$
h\left({ }^{\prime} \nabla_{\varphi_{v}} \varphi u, Y\right)=h\left(\phi \nabla_{v} u, Y\right) .
$$

As (7.7) holds for any $Y$, we have (7.3).

PROPOSITION 7.3. Let $\phi$ be an $(m-1)^{s}$-homothety of $M$ to $N$ such that $\beta$ is constant, then two following conditions are equivalent:

(i) \&y is parallel (along $D$ resp.).

(ii) ${ }^{\delta} \xi$ is parallel (along $\phi D$ resp.).

ProOF. (i) $\rightarrow$ (ii). We use (2.7). If $\zeta$ is parallel along $D, \nabla_{v} \zeta=0$ holds good provided that $v$ belongs to $D$, and we have ' $\nabla_{\varphi_{v}} \varphi \zeta=0$. Then $\sqrt{\alpha+\beta} \xi$, and so $\xi$, is parallel along $\varphi D$. If $\zeta$ is parallel, each trajectory of $\zeta$ is a geodesic. By Theorem 3.1 we see that each trajectory of $\xi$ is also a geodesic. Then $\xi$ is parallel field. The case (ii) $\rightarrow$ (i) reduces to the first case by taking the inverse $\phi^{-1}$.

THEOREM 7.4. Suppose that ' $\mathrm{y}$ is parallel along $D$ and $\phi$ is an $(m-1)^{s}$ homothety of $M$ to $N$. Let $u, v, r$ be vector fields which belong to $D$, then we have

$$
' R(\phi u, \phi v) \phi r=\varphi(R(u, v) r),
$$

where $R$ and ' $R$ denote the Riemannian curvature tensors with respect to $g$ and $h$.

PROOF. The expression of the Riemannian curvature tensor is as follows: 


$$
-R(u, v) r=\nabla_{u} \nabla_{v} r-\nabla_{v} \nabla_{u} r-\nabla_{[u, v]} r
$$

Thus, if $u, v, r$ belong to $D$, by Lemma 7.2 , we have

$$
\begin{aligned}
-{ }^{\prime} R(\varphi u, \varphi v) \phi r & ={ }^{\prime} \nabla_{\varphi u}{ }^{\prime} \nabla_{\varphi v} \varphi r-{ }^{\prime} \nabla_{\varphi v}{ }^{\prime} \nabla_{\varphi u} \varphi r-{ }^{\prime} \nabla_{[\varphi u, \varphi v]} \varphi r \\
& ={ }^{\prime} \nabla_{\varphi u} \varphi\left(\nabla_{v} r\right)-{ }^{\prime} \nabla_{\varphi v} \varphi\left(\nabla_{u} r\right)-{ }^{\prime} \nabla_{\varphi[u, v]} \varphi r \\
& =-\varphi \cdot R(u, v) r,
\end{aligned}
$$

completing the proof.

We denote by $K_{x}(u, v)$ the sectional curvature defined by the tangent vectors $u$ and $v$ at a point $x$, then

$$
K_{x}(u, v)=\frac{g_{x}(R(u, v) u, v)}{|u \wedge v|^{2}}
$$

where $|u \wedge v|$ is the area of the parallelogram with $u$ and $v$ as adjacent sides:

$$
|u \wedge v|^{2}=|u|^{2}|v|^{2}-(g(u, v))^{2} .
$$

REMARK 1. If ${ }^{\varepsilon} \zeta$ is a parallel field, we have $R_{j k l}^{i} w^{j}=0$. Therefore the sectional curvature $K(\zeta, u)$ determined by $\zeta$ and any other vector $u$ is equal to zero.

THEOREM 7.5. Assume that ${ }^{\circ} \zeta$ is parallel along $D$ and $\phi$ is an $(m-1)^{s}$ homothety. Let $u, v$ be tangent vectors at $x \in M$ which belong to $D_{x}$, then we have

$$
K_{x}(u, v)=\alpha^{\prime} K_{\varphi x}(\varphi u, \phi v)
$$

where 'K(фu, $\phi v)$ is the sectional curvature determined by $\phi u$ and $\phi v$ with respect to $h$.

PROOF. By Theorem 7.4, we have

$$
\begin{aligned}
h\left({ }^{\prime} R(\varphi u, \phi v) \phi u, \phi v\right) & =h(\phi \cdot R(u, v) u, \phi v) \\
& =\alpha g(R(u, v) u, v) \cdot \varphi^{-1} .
\end{aligned}
$$

From (7. 10), it follows that

$$
|\phi u \wedge \phi v|^{2}=\alpha^{2}|u \wedge v|^{2} \cdot \phi^{-1}
$$


By (7.12) and (7.13), we get (7.11).

q.e.d.

Let $u_{x}, v_{x}$ be two tangent vectors at $x$ and let $u, v$ be their extension to vector fields. Then the value of the function $K(u, v)$ at $x$ is equal to $K_{x}\left(u_{x}, v_{x}\right)$. Now we prove

THEOREM 7.6. Assume that ${ }^{\varepsilon} \xi$ and $\delta \xi$ are parallel fields and $\phi$ is an $(m-1)^{s}$-homothety. Then we have

$$
K(u, v)=\left(\alpha+\frac{Q(\varphi, u, v)}{|u \wedge v|^{2}}\right)^{\prime} K(\phi u, \phi v) \cdot \varphi
$$

for any vector fields $u$ and $v$, where we have put

$$
Q(\varphi, u, v)=\beta\left(a^{2} g(v, v)+b^{2} g(u, u)-2 a b g(u, v)\right),
$$

$a$ and $b$ denoting ${ }^{\varepsilon} w(u)$ and ${ }^{\varepsilon} w(v)$ respectively.

PROOF. We decompose $u$ and $v$ as follows:

$$
u=u_{0}+a \zeta, \quad v=v_{0}+b \zeta,
$$

where $u_{0}$ and $v_{0}$ belong to $D$ and $a={ }^{8} a={ }^{\varepsilon} w(u), b={ }^{\varepsilon} b={ }^{\varepsilon} w(v)$. Then $\phi u=\phi u_{0}$ $+\left({ }^{\varepsilon} a \varphi^{-1}\right) \varphi \zeta=\phi u_{0}+\left({ }^{\varepsilon} a \varphi^{-1}\right)^{\varepsilon \delta} \mu^{\delta} \xi, \mu^{2} \cdot \phi=\alpha+\beta$, and as $\zeta$ and $\xi$ are parallel fields, we have

$$
\begin{aligned}
h\left({ }^{\prime} R(\phi u, \phi v) \phi u, \phi v\right) & =h\left({ }^{\prime} R\left(\phi u_{0}, \phi v_{0}\right) \phi u_{0}, \phi v_{0}\right) \\
& =\alpha g\left(R\left(u_{0}, v_{0}\right) u_{0}, v_{0}\right) \cdot \phi^{-1} \\
& =\alpha g(R(u, v) u, v) \cdot \phi^{-1}
\end{aligned}
$$

On the other hand, using (7.10) and $\phi \zeta=\mu \xi, \mu^{2} \cdot \phi=\alpha+\beta$, we can show the following relation

$$
|\phi u \wedge \varphi v|^{2} \cdot \phi=\alpha^{2}|u \wedge v|^{2}+\alpha \beta\left(a^{2} g(v, v)+b^{2} g(u, u)-2 a b g(u, v)\right) .
$$

Thus we have

$$
\begin{aligned}
K(u, v) & =\left(\frac{1}{\alpha}\right) \frac{h\left(^{\prime} R(\varphi u, \varphi v) \varphi u, \phi v\right) \cdot \varphi}{|u \wedge v|^{2}} \\
& =\frac{1}{\alpha} \cdot \frac{\alpha^{2}|u \wedge v|^{2}+\alpha Q(\varphi, u, v)}{|u \wedge v|^{2}}\left({ }^{\prime} K(\phi u, \phi v) \cdot \varphi\right) .
\end{aligned}
$$


Now we have (7.14).

THEOREM 7.7. Assume that ${ }^{\varepsilon} \xi$ and ${ }^{\delta} \xi$ are parallel fields and $\varphi$ is an $(m-1)^{s}$-homothety. If $M$ is of non-negative curvature (non-positive curvature. respectively), then $N$ is of non-negative curvature (non-positive curvature respectively).

Proof. By (7.17) we see that $K(u, v)$ and ' $K(\varphi u, \varphi v)$ have the same sign + or - .

REMARK 2. In Theorem 7.6 and 7.7 , if $\phi$ is an $(m-1)^{s}$-homothety such that $\beta$ is constant, then the assumption that $\delta \xi$ is a parallel field may be removed by Proposition 7.3.

8. (m-1)-Einstein spaces. Let $M$ and $R_{1}$ be an $m$-dimensional Riemannian manifold and Ricci curvature.

Definition. If $M$ admits an $(m-1)$-dimensional distribution $D$ such that $R_{1}(u, v)=e g(u, v)$ holds good for $u, v \in D, e$ denoting a scalar field, we say that $M$ is an $(m-1)$-Einstein space with respect to the distribution $D$.

Let ${ }^{\varepsilon} \xi,{ }^{\varepsilon} w$ be ones defined in $\$ 1$. By the similar argument we see that $R_{1}$ is written as follows

$$
R_{1}=e g+{ }^{\varepsilon} w \otimes{ }^{\varepsilon} K+{ }^{\varepsilon} K \otimes{ }^{\varepsilon} w+f^{\varepsilon} w \otimes{ }^{\varepsilon} w
$$

where $f$ is a scalar field on $M$ and ${ }^{\varepsilon} K$ defines a 1 -form $K_{U}$ in each $U$ in $\$ 1$. Namely in $U$, we have

$$
R_{i j}=e g_{i j}+w_{i} K_{j}+K_{i} w_{j}+f w_{i} w_{j}
$$

where $K_{i} w^{i}=0$. Transvecting (8.2) with $g^{i j}$, we get

$$
R=m e+f .
$$

By the same letter $K$ we denote the contravariant vector: $K^{i}=g^{i j} K_{j}$. Using $\delta$, we have $(\delta w)=-w^{i},{ }_{i}$.

Now we prove

THEOREM 8.1. Suppose that $M$ is an $(m-1)$-Einstein space $(m>3)$ with respect to D. If in (8.1), the three conditions:

(1) $\delta w_{U}=0, \quad \nabla_{\xi_{U}} \zeta_{U}=0$, 
(2) $R_{1}\left(\zeta_{U}, \zeta_{U}\right)=$ constant

(3) $\delta K_{U}=0, \quad \nabla_{\zeta_{U}} K_{U}+\nabla_{K_{U}} \zeta_{U}=0$

are satisfied for each $U$, then $e$ and $f$ are constant on $M$. Further the scalar curvature $R$ is constant.

ProOF. Multiply $w^{i} w^{j}$ to (8.2) and contract with respect to $i$ and $j$, then we have

$$
R_{i j} w^{i} w^{j}=e+f
$$

Hence, by (2) we have

$$
e_{k}+f_{k}=0
$$

Differentiating covariantly (8.3) we have

$$
R_{, k}=m e_{k}+f_{k} .
$$

And from (8.2), we get

$$
g^{i s} R_{i k, s}=R_{i k}{ }^{i}=e_{k}+f_{i} w^{i} w_{k},
$$

where we have used $w^{i}{ }_{i}=0, w_{k, i} w^{i}=0, K^{i}{ }_{i}=0$ and $K_{k, i} w^{i}+w_{k, i} K^{i}=0$. Using the well-known identity $R_{, k}=2 R_{i k}{ }^{i},(8.6)$ and (8.7) show that

$$
(m-2) e_{k}=2 \zeta f \cdot w_{k}-f_{k} .
$$

Eliminating $f_{k}$ from (8.5) and (8.8), we have

$$
(m-3) e_{k}=2 \zeta f \cdot w_{k} .
$$

Transvecting (8.5) and (8.9) with $w^{k}$, we get

$$
\zeta e+\zeta f=0, \quad(m-3) \zeta e=2 \zeta f .
$$

Thus we get $(m-1) \zeta e=0$ and $\zeta e=0, \zeta f=0$. Then from (8.5), (8.6) and (8.9) it follows that $e_{k}=f_{k}=0$ and $R_{, k}=0$.

COROLLARY 8.2. In an (m-1)-Einstein space $(m>3)$, if $\varepsilon$ is a parallel field and if $\delta K_{U}=0, \nabla_{\xi_{U}} K_{U}=0$ (in particular if $K=0$ ) hold good. Then $e$, $f$ and $R$ are constant. 
ProOF. (1) of the Theorem holds good. By Ricci's indentity, we have $R_{i j} w^{i} w^{j}=0$, satisfying (2).

PROPOSITION 8.3. In the above Theorem, if $m=3$, i.e., $M$ is a (3-1)Einstein space satisfying (1), (2) and (3). Then

$$
\zeta e=\zeta f=0 \quad \text { and } \quad \zeta R=0 .
$$

Proof. By (8.9) we have $\zeta f=0$. And so $\zeta e=0$ and $\zeta R=0$ follow from (8.5) and (8.6).

DEFINITION. We call $M$ a ${ }^{\varepsilon} w$-Einstein space if $M$ is an $(m-1)$-Einstein space with respect to $D$ and satisfies ${ }^{\varepsilon} K=0$ in (8.1).

REMARK 1. In the study of contact manifolds, some authors treated with $\eta$-Einstein spaces, $\eta$ denoting a contact form ([11], [12]).

REMARK 2. In the Theorem 8.1 and Proposition 8.3, if $M$ is a ${ }^{\varepsilon} w$-Einstein space, the condition (3) is satisfied always.

If $M$ is an Einstein space $(R \neq 0)$, a transformation which preserves the Ricci curvature is an isometry of $M$. So there is no essentially $[m-1]$ conformal transformation of $M$ which preserves the Ricci curvature. This is one of the reasons why we consider $(m-1)$-Einstein spaces.

THEOREM 8.4. Let $M$ be an (m-1)-Einstein space. If a transformation $\varphi$ of $M$ preserves the Ricci curvature and the distribution ${ }^{\varepsilon} w=0$, then $\varphi$ is an $[m-1]$-conformal transformation.

PROOF. By assumption we have

$$
R_{1}(\phi u, \phi v)=e g(\phi u, \phi v)+w(\varphi u) K(\phi v)+K(\phi u) w(\phi v)+f w(\phi u) w(\phi v)
$$

for any vector fields $u, v$ on $M$. And we have a family ${ }^{\varepsilon \varepsilon^{\prime}} \boldsymbol{\gamma}=\left\{\boldsymbol{\gamma}_{U V}\right\}$ of scalar fields such that $\phi^{*} w=\gamma w$. As $R_{1}(\varphi u, \phi v) \cdot \varphi=R_{1}(u, v)$, we have

$$
\begin{aligned}
\phi^{*} g= & \frac{e}{e \cdot \phi} g+\frac{1}{e \cdot \phi} w \otimes\left(K-\gamma \phi^{*} K\right) \\
& \quad+\frac{1}{e \cdot \phi}\left(K-\gamma \phi^{*} K\right) \otimes w+\frac{1}{e \cdot \phi}\left(f-\gamma^{2}(f \cdot \phi)\right) w \otimes w .
\end{aligned}
$$


Though this is not a canonical form of an $(m-1)$-conformal transformation, we see that $\phi$ is an $[m-1]$-conformal transformation. If $K-\gamma \phi^{*} K$ is proportional to $w, \boldsymbol{\varphi}$ is an $[m-1]^{s}$-conformal transformation. And if $e$ is constant, $\varphi$ is an $[m-1]^{s}$-isometry.

COROLLARY 8.5. Let $M$ be $a^{\varepsilon}$ re-Einstein space $(m>3)$ and suppose that $\phi$ preserves the Ricci curvature and the distribution ${ }^{\varepsilon} w=0$. If (1) $\delta w_{I}=0$, $\nabla_{\xi_{U}} \zeta_{U}=0$ and (2) $R_{1}\left(\zeta_{U}, \zeta_{U}\right)$ is constant, then $\phi$ is an $[m-1]^{s}$-isometry.

ProOF. As $K=0$, by Theorem 8.1, we see that $e$ and $f$ are constant. Thus by (8.10), we get

$$
\phi^{*} g=g+\frac{f}{e}\left(1-\gamma^{2}\right) w \otimes w
$$

COROllaRy 8.6. In Corollary 8.5, in particular if $R_{1}\left(\zeta_{U}, \zeta_{U}\right)=$ non-zero constant. Then $\phi$ is an isometry.

PROOF. From $\phi^{*} w=\gamma_{w}, \varphi \zeta=\left(\gamma \cdot \phi^{-1}\right) \zeta$ follows. By contraction (8.11) with $\zeta$, we get

$$
\gamma^{2}=1+\frac{f}{e}\left(1-\gamma^{2}\right)
$$

As $e+f \neq 0$, we have $\gamma^{2}=1$, and hence $\phi^{*} g=g$.

9. The group of $[m-1]$-conformal transformations. In $m$-dimensional manifold $M$, let $D$ be an $(m-1)$-dimensional distribution of class $C^{\infty}$. By $\Pi$ we denote the set of all $[m-1]$-conformal transformation of $M$ on itself with respect to the distribution $D$. Let $\phi_{1}, \phi_{2}$ and $\phi_{3}$ be elements of $\Pi$, then

$$
\begin{gathered}
\boldsymbol{\varphi}_{\lambda}^{*} w=\gamma_{\lambda} w, \\
\left(\boldsymbol{\phi}_{\lambda}^{*} g\right)_{x}=\alpha_{\lambda}(x) g_{x}+w_{x} \otimes\left(\theta_{\lambda}\right)_{x}+\left(\theta_{\lambda}\right)_{x} \otimes w_{x}+\beta_{\lambda}(x) w_{x} \otimes w_{x},
\end{gathered}
$$

$\lambda=1,2,3$, where $\gamma_{\lambda}, \alpha_{\lambda}, \beta_{\lambda}$ are scalar fields and $\theta_{\lambda}$ defines 1 -form in each local neighborhood. Then the composition $\boldsymbol{\varphi}_{2} \cdot \boldsymbol{\varphi}_{1}$ satisfies

$$
\begin{aligned}
& \left(\left(\boldsymbol{\varphi}_{2} \cdot \boldsymbol{\varphi}_{1}\right)^{*} \boldsymbol{w}\right)_{x}=\left(\boldsymbol{\phi}_{1}{ }^{*} \boldsymbol{\varphi}_{2}{ }^{*} w\right)_{x}=\gamma_{1}(x) \gamma_{2}\left(\boldsymbol{\varphi}_{1} x\right) w_{x}, \\
& \left(\left(\boldsymbol{\varphi}_{2} \cdot \boldsymbol{\varphi}_{1}\right) * g\right)_{x}=\alpha_{1}(x) \alpha_{2}\left(\boldsymbol{\varphi}_{1} x\right) g_{x}
\end{aligned}
$$




$$
\begin{aligned}
& +w_{x} \otimes\left[\alpha_{2}\left(\phi_{1} x\right)\left(\theta_{1}\right)_{x}+\gamma_{1}(x)\left(\phi_{1}^{*} \theta_{2}\right)_{x}\right] \\
& +\left[\alpha_{2}\left(\phi_{1} x\right)\left(\theta_{1}\right)_{x}+\gamma_{1}(x)\left(\phi_{1}^{*} \theta_{2}\right)_{x}\right] \otimes w_{x} \\
& +\left[\alpha_{2}\left(\phi_{1} x\right) \beta_{1}(x)+\beta_{2}\left(\phi_{1} x\right) \gamma_{1}^{2}(x)\right] w_{x} \otimes w_{x},
\end{aligned}
$$

$$
\left(\left(\boldsymbol{\varphi}_{3} \cdot \boldsymbol{\varphi}_{2} \cdot \boldsymbol{\varphi}_{1}\right)^{*} g\right)=\alpha_{1}(x) \alpha_{2}\left(\boldsymbol{\varphi}_{1} x\right) \alpha_{3}\left(\boldsymbol{\varphi}_{2} \boldsymbol{\varphi}_{1} x\right) g_{x}+(\cdots)
$$

where $(\cdots)$ means three terms corresponding to the $2,3,4$ th term in the right hand side of (9.4). The inverse transformation of $\varphi$ satisfies

$$
\begin{gathered}
\left(\boldsymbol{\phi}^{-1 *} w\right)_{\phi x}=\left(\frac{1}{\gamma}\right)(x) w_{\phi x}, \\
\left(\boldsymbol{\phi}^{-1 *} g\right)_{\varphi x}=\left(\frac{1}{\alpha}\right)(x) g_{\varphi x}+w_{\varphi x} \otimes\left(-\frac{1}{\alpha \gamma}(x)\right)\left(\boldsymbol{\phi}^{-1 * \theta}\right)_{\varphi x} \\
+\left(-\frac{1}{\alpha \gamma}(x)\right)\left(\phi^{-1 *} \theta\right)_{\varphi x} \otimes w_{\varphi x}-\left(\frac{\beta}{\alpha \gamma^{2}}\right)(x) w_{\varphi x} \otimes w_{\varphi x} .
\end{gathered}
$$

Here we notice that (9.4) and (9.7) are not canonical expression of $(m-1)$ conformal transformations.

We use the notations for the subgroups of the transformation group $\Pi$ as follows :

$\Pi^{s}$ : The totality of $[m-1]^{s}$-conformal transformations.

$\Theta$ : The totality of $[m-1]$-homotheties.

$\Phi$ : The totality of $[m-1]$-isometries.

$\Theta^{s}: \Theta \cap \Pi^{s}, \quad \Phi^{s}: \Phi \cap \Pi^{s}$.

Next theorem is an immediate consequence of (9.5) and (9.7).

THEOREM 9.1. $\Phi$ and $\Phi^{s}$ are normal subgroups of $\Theta$ and $\Theta^{s}$ respectively.

THEOREM 9.2. Any finite subgroup of $\Theta$ is a subgroup of $\Phi$.

ProOF. Let $\phi \in \Theta$, then by (9.4) we have

$$
\left(\phi^{2 *} g\right)_{x}=\alpha^{2} g_{x}+(*)
$$

and by $k$ times iterations we have

$$
\left(\phi^{k *} g\right)_{x}=\alpha^{k} g_{x}+\left({ }^{* *}\right),
$$


where $(*)$ and $(* *)$ denote the terms which contain $w_{x}$. So, as $\alpha^{k}$ is not bounded unless $\alpha=1$, the assertion is true.

q.e.d.

Some answers to the question "Under what conditions does certain subgroup of $\Pi^{s}$ make a Lie group ?” are given in $\$ 15$.

10. $(m-1)$-conformal transformations of complete or compact $M$. Let $\phi$ be an $(m-1)$-conformal transformation of $M$ onto itself satisfying (1.6) or (4.1). We take an arbitrary point $x$ of $M$ and take suitable local coordinates $x^{i}$ in a local coordinate neighborhood $U$ about $x$ such that $\left(g_{i j}\right)_{x}=\delta_{i j}, w_{x}=(0$, $\cdots, 0,1)$. This is possible as $\zeta_{U}$ is a unit vector field. And let $\left(\theta_{1}, \cdots, \theta_{n-1}, 0\right)$ be components of $\theta_{U}$, where we have used $\theta_{m}=0$ as $\theta(\xi)=0$. Then we have

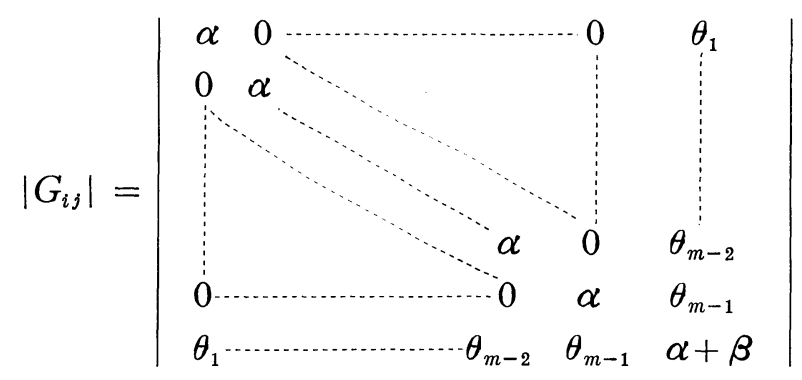

where $\left|G_{i j}\right|$ denotes the determinant of the matrix $G_{i j}$. Thus

$$
\left|G_{i j}\right|=\alpha^{m-1}\left\{(\alpha+\beta)-\frac{1}{\alpha} \sum_{i=1}^{m-1} \theta_{i}^{2}\right\}
$$

holds at $x$. As $\sum_{i=1}^{m-1} \theta_{i}^{2}=g(\theta, \theta)$ and $\left|G_{i j}\right|,\left|g_{i j}\right|$ are positive, we get

$$
\sqrt{\left|G_{i j}\right|}=\left[\alpha^{m-1}\left(\alpha+\beta-\alpha^{-1} g(\theta, \theta)\right)\right]^{\frac{1}{2}} \sqrt{\left|g_{i j}\right|} .
$$

If $M$ is compact and orientable, we can integrate (10.1) over $M$, denoting $\int_{M} d \sigma=|M|$, we have

THEOREM 10.1. Suppose that $\boldsymbol{\phi}$ is an (m-1)-conformal transformation of a compact and orientable manifold $M$ onto itself. Then the following equation is valid:

$$
\frac{1}{|M|} \int_{M}\left[\alpha^{m-1}\left(\alpha+\beta-\alpha^{-1} g(\theta, \theta)\right)\right]^{\frac{1}{2}} d \sigma=1
$$


As an immediate consequence of Theorem 10.1, we get

THEOREM 10.2. Let $\boldsymbol{\phi}$ be an $(m-1)^{s}$-homothety of a compact and orientable manifold onto itself such that $\beta$ is constant. Then $\phi$ is an isometry, except the case $\alpha \neq 1$ and $\beta=\alpha^{1-m}-\alpha$.

As a corollary we have

COROLLARY 10.3. In a compact orientable manifold, (i) if $\phi$ is an $(m-1)^{s}$-homothety such that $\phi^{\varepsilon} \zeta= \pm^{\varepsilon} \zeta$, then $\phi$ is an isometry. (ii) if $\phi$ is an $(m-1)^{s}$-isometry such that $\beta$ is constant, then $\phi$ is an isometry.

Next we prove

THEOREM 10.4. Let $\boldsymbol{\rho}$ be an $(m-1)^{s}$-conformal transformation of a complete Riemannian manifold $M$. If $\alpha<\alpha_{0}<1$ and $\alpha+\beta<\alpha_{0}<1$ on $M$ (or $\alpha>\alpha_{0}>1$ and $\alpha+\beta>\alpha_{0}>1$ ) for some constant $\alpha_{0}$, then there exists $a$ unique fixed point of $\boldsymbol{\phi}$ in $M$.

PROOF. Let $x$ be an arbitrary point of $M$ and $l=x(t)(0 \leqq t \leqq 1)$ be any differentiable curve which joins $x=x(0)$ and $x(1)=\phi x$. We denote by $|l|$ the length of $l$. Now we have

$$
g_{\varphi x(t)}\left(\varphi \frac{d x}{d t}, \varphi \frac{d x}{d t}\right)=\alpha_{x(t)} g\left(\frac{d x}{d t}, \frac{d x}{d t}\right)+\beta_{x(t)}\left(w\left(\frac{d x}{d t}\right)\right)^{2}
$$

We decompose $\frac{d x}{d t}$ as $\frac{d x}{d t}=v_{t}+r_{t} \zeta, v_{t} \in D_{x(t)}$, then

$$
\begin{aligned}
g_{\varphi x(t)}\left(\varphi \frac{d x}{d t}, \varphi \frac{d x}{d t}\right) & =\alpha_{x(t)}\left(g\left(v_{t}, v_{t}\right)+r_{t}^{2}\right)+\beta_{x(t)} r_{t}^{2} \\
& =\alpha_{x(t)}\left(v_{t}, v_{t}\right)+(\alpha+\beta)_{x(t)} r_{t}^{2} \\
& <\alpha_{0} g\left(\frac{d x}{d t}, \frac{d x}{d t}\right)
\end{aligned}
$$

Thus the length $|\phi l|$ of $\phi l$ is smaller than $|l|$. By iteration we get $\left|\phi^{k} l\right|$ $\left\langle\alpha_{0}^{k}|l|\right.$ for any integer $k$. Therefore $\left(x, \phi x, \cdots, \phi^{k} x, \cdots\right)$ is a Cauchy sequence. By completeness of $M$, we have limit point $\bar{x}: \varphi \bar{x}=\bar{x}$.

In the case $\alpha>\alpha_{0}>1$ and $\alpha+\beta>\alpha_{0}>1$, we have $\left|\phi^{k} l\right|>\alpha^{k}|l|$. Thus this case reduces to the first case by consideration of $\phi^{-1}$. Uniqueness of $\bar{x}$ is seen as follows: If there exist two fixed points $\bar{x}, \bar{x}^{\prime}$ of $\varphi$, we can join $\bar{x}$ 
and $\bar{x}^{\prime}$ by the shortest curve $l^{\prime}$, then $\phi l^{\prime}$ is of the smaller length than $\left|l^{\prime}\right|$ which is a contradiction.

COROLlARY 10.5. Suppose that ${ }^{\varepsilon} \zeta$ is a parallel field and let $\boldsymbol{\varphi}$ be an $(m-1)^{s}$-homothety of a complete Riemannian manifold $M$ such that $\beta$ is constant satisfying $\alpha<1$ and $\alpha+\beta<1$ (or $\alpha>1$ and $\alpha+\beta>1$ ). Then $M$ is (locally) Euclidean.

PROOF. By Theorem 4.2, $\boldsymbol{\rho}$ is an affine transformation of $M$. And by Proposition 10.4, $\phi$ has a fixed point $\bar{x}$. Then by [2], or [5], $M$ is locally Euclidean.

11. Supplimentary results. (i) Space of constant curvature. A manifold $M$ is said to be constant curvature if the Riemannian curvature $R$ satisfies

$$
R(u, v) z=\kappa\{g(v, z) \cdot u-g(u, z) \cdot v\}
$$

for any vector fields $u, v, z$ on $M$, where $\kappa$ is constant.

THEOREM 11.1. Let $M$ be of constant curvature and $\boldsymbol{\varphi}$ be $(m-1)^{s}$ conformal transformation of $M$ onto $M$. If ${ }^{\varepsilon} w(z)=0$, then we have

$$
R(\phi u, \phi v) \phi z=\phi(\alpha R(u, v) z) \text {. }
$$

Proof. By (11.1), we get

$$
\begin{aligned}
R(\boldsymbol{\varphi} u, \boldsymbol{\varphi v}) \boldsymbol{\phi} z & =\kappa\{g(\boldsymbol{\varphi} v, \boldsymbol{\varphi z}) \boldsymbol{\varphi} u-g(\boldsymbol{\varphi} u, \varphi z) \boldsymbol{\varphi} v\} \\
& =\boldsymbol{\phi}(\alpha R(u, v) z)
\end{aligned}
$$

because $g(\boldsymbol{\phi} v, \boldsymbol{\phi} z)=\alpha g(v, z) \cdot \boldsymbol{\phi}^{-1}$.

(ii)

THEOREM 11.2. We assume that $\left.R_{1}{ }^{\circledR}{ }^{\natural} \zeta,{ }^{\varepsilon} \zeta\right)=T$ and the scalar curvature $R$ are constant and $R \neq T$. If $\phi \in \Pi^{s}$ leaves $R_{1}$ invariant, then $\phi \in \Phi^{s}$. Further if $T \neq 0, \varphi$ is an isometry of $M$.

PROOF. As $T$ is constant and $\varphi \xi=\mu \xi, \mu^{2} \cdot \varphi=\alpha+\beta$, we have

$$
T=R_{1}(\phi \zeta, \phi \zeta)=(\alpha+\beta) T
$$

On the other hand, we have 


$$
R={ }^{\Phi} R=\left(G^{-1}\right)^{i j} R_{i j}=\frac{1}{\alpha} R-\frac{\beta}{\alpha(\alpha+\beta)} T .
$$

Namely, we have

$$
(\alpha+\beta)(\alpha-1) R+\beta T=0
$$

We add $-(\alpha+\beta-1) T=0$ to the last equation, getting

$$
(\alpha+\beta)(\alpha-1) R-(\alpha-1) T=0 .
$$

If we use again (11.5), the last equation turns to $(\alpha+\beta)(\alpha-1)(R-T)=0$. So $\alpha=1$ follows. Furthermore, if $T \neq 0, \beta=0$ follows from (11.6).

\section{Chapter II}

12. Infinitesimal $(m-1)$-conformal and $[m-1]$-conformal transformations. Let $D$ be an $(m-1)$-dimensional distribution and $\varphi_{t}(|t|<q$; for some positive number $q$ ) be a local 1-parameter subgroup of $\Pi$, then we have

$$
\begin{gathered}
\boldsymbol{\varphi}_{t}{ }^{*} \varepsilon_{w} w=\gamma_{t}{ }^{\varepsilon} w \\
\boldsymbol{\varphi}_{t}^{*} g=\alpha_{t} g+{ }^{\varepsilon} w \otimes^{\varepsilon} \theta_{t}+{ }^{\varepsilon} \theta_{t} \otimes^{\varepsilon} w+\beta_{t}{ }^{\varepsilon} w \otimes^{\varepsilon} w
\end{gathered}
$$

for $t:|t|<q$. In this section too, we abbreviate frequently $\varepsilon$ in ${ }^{\varepsilon} z$ or ${ }^{\varepsilon} \theta$. As $\boldsymbol{\varphi}_{0}(t=0)$ is an identical transformation of $M$, we have

$$
L(v) w=\lim _{t \rightarrow 0} \frac{\gamma_{t}-1}{t} w
$$

(12.4) $L(v) g=\lim _{t \rightarrow 0} \frac{\alpha_{t}-1}{t} g+w \otimes\left(\lim _{t \rightarrow 0} \frac{\theta_{t}}{t}\right)+\left(\lim _{t \rightarrow 0} \frac{\theta_{t}}{t}\right) \otimes w+\lim _{t \rightarrow 0} \frac{\beta_{t}}{t} w \otimes w$,

where $v$ is a vector field on $M$ defined by $\boldsymbol{\phi}_{t}$. From these, we define that an infinitesimal transformation $u$ is an infinitesimal [m-1]-conformal transformation if it satisfies

$$
\begin{gathered}
L(u) w=c w \\
(L(u) g)(r, s)=0
\end{gathered}
$$

for any vector fields $r, s$ which belong to $D$. In (12.5), $c$ does not depend on 
the choice of $U$, so $c$ is a scalar field.

By the similar fashion to $\S 1$, we see that $L(u) g$ is written as

$$
L(u) g=a g+w \otimes F+F \otimes w+b w \otimes w,
$$

where $a$ and $b$ are scalar fields, and $\left.F={ }^{8} F\right)$ defines a 1-form $F_{V}$ in each neighborhood $U$ in such a way that $w_{U}$ and $F_{U}$ are orthogonal. When we use the local coordinates $x^{i}$ in $U, w$ and $F$ are treated as covariant tensors. If $F=0, v$ is called an infinitesimal $[m-1]^{s}$-conformal transformation. If $a$ is constant, $v$ is called an infinitesimal [ $m-1]$-homothetic transformation, etc.. But in many cases, we consider infinitesimal transformations which satisfy only (12.6), and we denote them by infinitesimal $(m-1)$-conformal transformation.

THEOREM 12.1. Let $u$ be an infinitesimal $[m-1]$-conformal transformation. Then $L(u)^{\varepsilon} \xi=p^{\varepsilon} \xi$ holds good for some scalar field $p$ if and only if $F=0$, i.e., $u$ in an infinitesimal $[m-1]^{s}$-conformal transformation. And we have $-2 p=2 c=a+b$. get

PROOF. Operating Lie differentiation to $w_{j}=w^{i} g_{i j}$ with respect to $u$, we

$$
c w_{j}=\left(L(u) w^{i}\right) g_{i j}+(a+b) w_{j}+F_{j} .
$$

If $L(u) w^{i}=p w w^{i}$, we get $F_{j}=0$. Conversely if $F_{j}=0$, transvecting the last equation with $g^{j k}$, we obtain

$$
L(u) w^{i}=(c-a-b) w^{i} .
$$

THEOREM 12.2. If $\zeta_{U}$ is an infinitesimal [m-1]-conformal transformation in each $U$. Then it is an infinitesimal $[m-1]^{s}$-conformal transformation in each $U$ and each trajectory of ${ }^{\circ} \zeta$ is a geodesic.

PROoF. By the equation $L(\zeta) w_{i}=c w_{i}$, we see that

$$
w_{i, j} w^{j}=c w_{i} .
$$

Transvecting the last equation with $w^{i}$, we get $c=0$ and $w_{i, j} w^{j}=0$. This means that each trajectory of $\zeta$ is a geodesic. Next as $L(\zeta) g=w_{i, j}+w_{j, i}$, we get

$$
w_{i, j}+w_{j, i}=a g_{i j}+w_{i} F_{j}+F_{i} w_{j}+b w_{i} w_{j} .
$$


Multiplying (12.8) by $w^{i} w^{j}$ and contracting, we have $a+b=0$. If we transvect (12.8) with $w^{j}$ and use $w_{i, j} w^{j}=0$, we have $(a+b) w_{i}+F_{i}=0$. Thus $F_{i}=0$. q.e.d.

In the above proof, we see also the following

THEOREM 12.3. If $\zeta_{U}$ is an infinitesimal $(m-1)$-conformal transformation in each $U$. And if each trajectory of ${ }^{\zeta}$ is a geodesic, then $\zeta_{U}$ is an infinitesimal $[m-1]^{s}$-conformal transformation and satisfie $a+b=0$.

Furthermore we have

THEOREM 12.4. If $\zeta_{U}$ is an infinitesimal $(m-1)$-conformal transformation and satisfies $\delta w_{U}=0$. Then it is an infinitesimal $(m-1)$-isometry in each $U$.

PROOF. Transvecting (12.8) with $w^{i} w^{j}$ and $g^{i j}$, we have $a+b=0$ and $m a+b=0$. Thus $a=0$ and $b=0$ hold good.

THEOREM 12.5. If $\zeta_{U}$ is an infinitesimal $(m-1)$-conformal transformation in each $U$. And if each trajecoty of $\zeta_{\sigma}$ is a geodesic and $\delta w_{\sigma}=0$. Then $\zeta_{U}$ is an infinitesimal isometry.

ProOF. By Theorem 12.4, we have $a=b=0$. On the other hand by Theorem 12.3, we have $F_{j}=0$ completing the proof.

THEOREM 12.6. Suppose that $\zeta_{U}$ be an infinitesimal $(m-1)$-conformal transformation, then $\rho \xi_{U}$ is also an infinitesimal $(m-1)$-conformal transformation for any scalar field $\rho$.

PROOF. First we have

$$
\left(\rho w_{i}\right)_{, j}+\left(\rho w_{j}\right)_{, i}=\rho\left(w_{i, j}+w_{j, i}\right)+\rho_{i} w_{j}+\rho_{j} w_{i} .
$$

On the other hand, $\rho_{i}$ is written as

$$
\rho_{j}=\left(\rho_{j}-\zeta \rho \cdot w_{j}\right)+\zeta \rho \cdot w_{j}
$$

Therefore, from (12.8) we get

$$
\begin{aligned}
L(\rho \zeta) g_{i j}= & a \rho g_{i j}+w_{i}\left(\rho F_{j}+\rho_{j}-\zeta \rho \cdot w_{j}\right)+\left(\rho F_{i}+\rho_{i}-\zeta \rho \cdot w_{i}\right) w_{j} \\
& +(b \rho+2 \zeta \rho) w_{i} w_{j} .
\end{aligned}
$$

This completes the proof. 
Conversely, we have

THEOREM 12.7. If $\rho \zeta_{U}$ is an infinitesimal $(m-1)$-conformal transformation for some non-vanishing scalar $\rho$. Then $\zeta_{U}$ is also an infinitesimal $(m-1)$-conformal transformation.

PROOF. We refer to the proof of Theorem 12.6.

Now let $u$ and $\rho u$ be two infinitesimal $(m-1)$-conformal transformations, then we have

$$
\begin{gathered}
u_{i, j}+u_{j, i}=a g_{i j}+w_{i} F_{j}+F_{i} w_{j}+b w_{i} w_{j}, \\
\left(\rho u_{i}\right)_{, j}+\left(\rho u_{j}\right)_{, i}=a^{\prime} g_{i j}+w_{i} F_{j}{ }_{j}+F_{i}^{\prime} w_{j}+b^{\prime} w_{i} w_{j},
\end{gathered}
$$

where $a, a^{\prime}, b, b^{\prime}$, are scalar fields. Subtracting (12.9) multiplied by $\rho$ from (12.10), we get

$$
\rho_{j} u_{i}+\rho_{i} u_{j}=\left(a^{\prime}-\rho a\right) g_{i j}+w_{i}\left(F_{j}^{\prime}-\rho F_{j}\right)+\left(F_{i}^{\prime}-\rho F_{i}\right) w_{j}+\left(b^{\prime}-\rho b\right) w_{i} w_{j}
$$

If $m>2$, there exists a vector field which is orthogonal to $u$ and $\zeta$, thus $a^{\prime}-\rho a=0$ follows form (12.11). Transvecting (12.11) with $w^{i} w^{j}$ and $g^{i j}$ respectively, we get

$$
\begin{aligned}
& 2 w(u) \zeta \rho=b^{\prime}-\rho b, \\
& 2 u \rho=b^{\prime}-\rho b,
\end{aligned}
$$

from which we get $u \rho=w(u) \zeta \rho$. Next transvecting (12.11) with $u^{i}$ and $w^{i}$ respectively, we have

$$
\begin{gathered}
u \rho \cdot u_{j}+\left(u_{i} u^{i}\right) \rho_{j}=w(u)\left(F_{j}^{\prime}-\rho F_{j}\right) \\
+\left(F_{i}^{\prime}-\rho F_{i}\right) u^{i} w_{j}+\left(b^{\prime}-\rho b\right) w(u) w_{j}, \\
\zeta \rho \cdot u_{j}+w(u) \rho_{j}=\left(F_{j}^{\prime}-\rho F_{j}\right)+\left(b^{\prime}-\rho b\right) w_{j} .
\end{gathered}
$$

Subtracting (12.13) multiplied $w(u)$ from (12.12) and using $u \rho=w(u) \zeta \rho$, we get

$$
\left(u_{i} u^{i}-w^{2}(u)\right) \rho_{j}=\left(F_{i}^{\prime}-\rho F_{i}\right) u^{i} w_{j} .
$$


THEOREM 12.8. Two different infinitesimal $(m-1)^{s}$-conformal transformations, both of which are not proportional to ${ }^{\circ} \zeta$ almost everywhere in $M$, cannot have the same streamlines, if $m \geq 3$.

PROOF. In (12.14), as $F$ and $F^{\prime}$ vanish, we have $\left(u_{i} u^{i}-w^{2}(u)\right) \rho_{j}=0$. That $u^{i}$ is not proportional to $w^{i}$ almost everywhere means, as usual, that the set of the point where $u^{i}$ is proportional to $w^{i}$ is of measure zero. And $u^{i}$ is proportional to $w^{i}$ at a point $x$ of $M$ if and only if $u_{i} u^{i}=w^{2}(u)$ at $x$. Thus we have $\rho_{j}=0$ almost everywhere, and hence everywhere on $M$. This means that $\rho$ is constant.

13. Lie derivative of the Christoffel's symbols by an infinitesimal $(m-1)$-conformal transformation and relations with an infinitesimal affine transformation and projective transformation. Let $u$ be an infinitesimal $(m-1)$-conformal transformation:

$$
L(u) g_{i j}=a g_{i j}+w_{i} F_{j}+F_{i} w_{j}+b w_{i} w_{j} .
$$

Into the following formula (see [22], p. 52)

$$
2 L(u)\left\{\begin{array}{l}
i \\
j k
\end{array}\right\}=g^{i r}\left(\nabla_{j} L(u) g_{r k}+\nabla_{k} L(u) g_{r j}-\nabla_{r} L(u) g_{j k}\right),
$$

we substitute (13.1), then we have

$$
\begin{aligned}
2 L(u)\left\{\begin{array}{l}
i \\
j k
\end{array}\right\}= & a_{j} \delta_{k}^{i}+a_{k} \delta_{j}^{i}-a^{i} g_{j k}+b_{j} w^{i} w_{k}+b_{k} w^{i} w_{j}-b^{i} w_{j} w_{k} \\
& +b\left\{w_{k}\left(w^{i}, j-w_{j}{ }^{i}\right)+w_{j}\left(w^{i}{ }_{, k}-w_{k}{ }^{i}\right)+w^{i}\left(w_{j, k}+w_{k, j}\right)\right\} \\
& +w^{i}\left(F_{j, k}+F_{k, j}\right)+\left(F^{i},{ }_{j}-F_{j}{ }^{i}\right) w_{k}+\left(F^{i},{ }_{, k}-F_{k}{ }^{, i}\right) w_{j} \\
& +F^{i}\left(w_{j, k}+w_{k, j}\right)+\left(w^{i}, j-w_{j}{ }^{, i}\right) F_{k}+\left(w^{i}, k-w_{k}{ }^{i}\right) F_{j} .
\end{aligned}
$$

Analogously to Theorem 4.1, we prove

THEOREM 13.1. Let $u$ be an infinitesimal $(m-1)^{s}$-conformal transformation. If $u$ is an infinitesimal affine transformation, then we have

(1) $a$ and $b$ are constant.

And as a necessary condition that $M$ admits such $u$ satisfying $b \neq 0$, we have

(2) ${ }^{\circ}$ is a parallel field.

PROOF. Transvecting (13.3) with $w^{k} w_{i}, \delta_{i}^{k}$ respectively and utilizing $F=0$, we get 


$$
\begin{aligned}
a_{j}+b_{j} & =0, \\
m a_{j}+b_{j} & =0 .
\end{aligned}
$$

Then we see that $a$ and $b$ are constant. Next we transvect (13.3) with $w^{s} w^{k}$ and, noticing $a_{j}=b_{j}=0$, we get $b w^{i}{ }_{j} w^{j}=0$. Transvecting (13.3) with $w^{j}$ and $w_{i}$ respectively, we have $b\left(w_{i, k}-w_{k, i}\right)=0$ and $b\left(w_{i, k}+w_{k, i}\right)=0$. Thus $w_{i}$ is a parallel field, if $b \neq 0$.

Conversely the following Theorem is obvious by (13.3).

THEOREM 13.2. If ${ }^{\text {¿ }}$ is a parallel field and $u$ is an infintiesimal $(m-1)^{s}$ homothetic transformation such that $b$ is constant. Then $u$ is an infinitesimal affine transformation.

An infinitesimal projective transformation $u$ is characterized by

$$
2 L(u)\left\{\begin{array}{l}
i \\
j k
\end{array}\right\}=2 \delta_{j}^{i} \bar{\psi}_{k}+2 \delta_{k}^{i} \bar{\psi}_{j}
$$

where $\bar{\psi}$ is a scalar field on $M$.

Analogously to Theorem 5.2 , we prove

THEOREM 13.3. If $u$ is an infinitesimal $(m-1)^{s}$-homothetic transformation and at the same time infinitesimal projective transformation. Then $u$ is an infinitesimal affine transformation. Further $b$ is constant and $\xi \xi$ is a parallel field.

PROOF. Transvecting (13.6) with $\delta_{i}^{k}, w^{k} w_{i}$ and using (13.3) with $F=0$, we have

$$
\begin{gathered}
2(m+1) \bar{\psi}_{j}=b_{j}, \\
2 \zeta \bar{\psi} \cdot w_{j}+2 \bar{\psi}_{j}=b_{j} .
\end{gathered}
$$

From (13.7) and (13.8) we deduce the relations $\zeta b=0$ and $\zeta \bar{\psi}=0$. Then it is easy to see that $\bar{\psi}_{j}$ vanishes. That $\zeta$ is a parallel field follows from Theorem 13.1.

14. Lie derivative of the Riemannian curvature tensor by an infinitesimal $(\boldsymbol{m}-\mathbf{1})$-conformal transformation. If we substitute (13.3) into the following formula ([22], p. 17) 


$$
2 L(u) R_{j k l}^{i}=2 \nabla_{l} L(u)\left\{\begin{array}{l}
i \\
j k
\end{array}\right\}-2 \nabla_{k} L(u)\left\{\begin{array}{l}
i \\
j l
\end{array}\right\},
$$

we get

$$
\begin{aligned}
& 2 L(u) R_{j k l}^{i}=2 a_{j,[l} \delta_{k^{\lrcorner}}^{i}-2 a_{,[l}^{i} g_{k] j}+2 w^{i} b_{j,[l} w_{k]}+2 w_{j} w_{[l} b_{, k]}^{i} \\
& +2 b_{j}\left(w_{,[l}^{i} w_{k]}+w^{i} w_{[k, l]}\right)+2 b^{i}\left(w_{j,[k} w_{l]}+w_{j} w_{[l, k]}\right) \\
& \left.+2 b_{\left[_{l}\right.}\left\{w_{k]}\left(w^{i}, j-w_{j}{ }^{i}\right)-w_{k]}{ }^{i} w_{j}+w_{k], j} w^{i}\right)\right\} \\
& +b\left\{2 w_{[k, l]}\left(w^{i}, j-w_{j}{ }^{i}\right)+2\left(w^{i}, j\left[l-w_{j}{ }^{i}{ }_{[l}\right) w_{k]}\right.\right. \\
& +2 w_{j,[k} w_{l]}{ }^{i}-2 w_{\left[k^{\prime} l\right]}{ }^{i} w_{j}+2 w^{i}{ }_{[l} w_{k], j}+2 w^{i} w_{[k,|j| l]} \\
& \left.+R_{r k l}^{i} w^{r} w_{j}-w^{i} R^{r}{ }_{j k l} w_{r}\right\}
\end{aligned}
$$

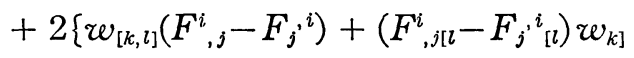

$$
\begin{aligned}
& +w_{j,[l}\left(F_{, k]}^{i}-F_{k]^{i}}{ }^{i}\right)+w_{j}\left(F^{i}{ }_{, k k l]}-F_{\left[k^{\prime}{ }^{i} l\right]}\right) \\
& +w_{[l}^{i}\left(F_{k], j}+F_{|j|, k]}\right)+w^{i}\left(F_{[k, \mid j[l]}+F_{j,[k l]}\right) \\
& +F_{[k, l]}\left(w_{, j}^{i}-w_{j}{ }^{i}\right)+\left(w_{, j[l}^{i}-w_{j}{ }^{i}{ }_{[l}\right) F_{k]} \\
& +F_{j,[l}\left(w^{i}{ }_{, k]}-w_{k]}{ }^{i}\right)+F_{j}\left(w^{i}{ }_{[k k l]}-w_{\left[k^{\prime}{ }^{i} l\right]}\right) \\
& \left.+F^{i}{ }_{, l l}\left(w_{k], j}+w_{|j|, k]}\right)+F^{i}\left(w_{[k,|j| l]}+w_{j,[k l]}\right)\right\} .
\end{aligned}
$$

Contracting with respect to $i$ and $l$, we have

$$
\begin{aligned}
& 2 L(u) R_{j k}=(2-m) a_{j, k}-a^{r}{ }_{, r} g_{j k}+2 \xi b \cdot w_{(j, k)} \\
& +2 w^{r}{ }_{, r} b_{(j} w_{k)}+2 w^{r}\left(b_{r,(j} w_{k)}+b_{(j} w_{k), r}\right) \\
& -2 b^{r}\left(2 w_{(j} w_{k), r}-w_{r,{ }_{j}} w_{k)}\right)-b_{j, k}-b_{, r}^{r} w_{j} w_{k} \\
& +2 b\left\{w^{r}{ }_{, r} w_{(j, k)}+w_{(j,}{ }^{r}\left(w_{|r|, k)}-w_{k), r}\right)\right. \\
& \left.\left.+w_{(j}\left(w^{r}, k\right) r-w_{k)}{ }^{r} r\right)+w_{(j, k) r} w^{r}\right\} \\
& +2 F^{r}{ }_{, r} w_{(j, k)}+2 w^{r}{ }_{, r} F_{(j, k)}+2 w^{r}{ }_{, k}\left(F_{j), r}-2 F_{|r|, j)}\right) \\
& \left.+2\left(F^{r}{ }_{(j}-2 F_{(j}{ }^{r}\right) w_{k), r}+2 w_{(k}\left(F^{r}, j\right) r-F_{j)}{ }_{r}{ }_{r}\right) \\
& \left.+2 F_{(k}\left(w^{r}, j\right) r-w_{j)}{ }^{r} r\right)+2 w_{(j, k) r} F^{r}+2 F_{(j, k) r} w^{r}+4 w^{r}{ }_{, j} F_{|r|, k)} .
\end{aligned}
$$

On the other hand, we have

$$
L(u) g^{j k}=-a g^{j k}-w^{j} F^{k}-F^{j} w^{k}-b w^{j} w^{k},
$$




$$
L(u) R=L(u) g^{j k} \cdot R_{j k}+g^{j k} L(u) R_{j k} .
$$

Transvecting (14.3) with $g^{j k}$, and substituting the result into (14.5), we have after calculation

$$
\begin{aligned}
& L(u) R=-a R-2 R_{j k} w^{j} F^{k}-b R_{j k} w^{j} w^{k}+(1-m) a^{r}, r \\
& -b^{r}{ }_{, r}+\left\{2 \zeta b \cdot w^{r}, r+\zeta(\zeta b)+w_{j, k} b^{j} w^{k}+b\left(w^{r}, r\right)^{2}\right. \\
& +b\left(w^{r, l} w_{l, r}+w^{r} w^{l, r l}+w^{r} w_{, l r}^{l}\right)
\end{aligned}
$$

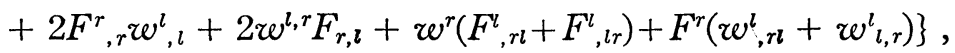

where we have used $0=\left(w^{k} F_{k}\right)_{, r}{ }^{r}=w^{k, r}{ }_{r} F_{k}+2 w^{k, r} F_{k, r}+w^{k} F_{k, r} r$.

We sometimes write $F_{U}$ to denote not only 1 -form but also for a contravariant vector field on $U$ associated with it. And ${ }^{\varepsilon} F=\left\{F_{U}\right\}$.

PROPOSITION 14.1. Suppose that $u$ is an infinitesimal $(m-1)$-conformal transformation on $M$. Then we have

$$
\left.L(u) R+a R+2 R_{1}\left({ }^{\varepsilon} \zeta,{ }^{\varepsilon} F\right)+b R_{1}{ }^{\varepsilon} \xi,{ }^{\varepsilon} \zeta\right)=\delta\left(u,{ }^{\varepsilon} \zeta\right)
$$

where $(u, \xi \xi)$ denotes a certain 1-form on $M$.

PROOF. The sixth term indicated by \{\} of the right hand side of (14.6) is equal to

$$
\begin{aligned}
& \left(\zeta b \cdot w^{r}\right)_{, r}+\left(b w^{r} w_{, r}^{l}\right)_{, l}+\left(b w^{l} w^{r}, r\right)_{, l} \\
& \quad+\left(w^{l} F^{r}, r\right)_{, l}+\left(F^{r} w_{, l}^{l}\right)_{, r}+\left(w^{r} F_{, r}^{l}\right)_{, l}+\left(F^{r} w_{, r}^{l}\right)_{, l} .
\end{aligned}
$$

Although $\zeta, F$ and $w$ are generally neither globally defined vector field nor 1 -forms, each term of the above (*) contains two of $\zeta, F, w$. Thus each term can be considered as a $\delta$-image of a globally defined 1-form. As $a$ and $b$ are scalar fields, we have (14.7) from (14.6).

PROPOSITION 14.2. Suppose that $\zeta_{U}$ is an incompressible vector field on each $U$ and each trajectory of $\zeta_{U}$ is a geodesic. Then an infinitesimal $(m-1)^{s}$-conformal transformation $u$ on $M$ satisfies

$$
L(u) R=-a R-b R_{1}\left(\zeta_{U}, \zeta_{U}\right)-(1-m) \delta d a+\zeta_{v}\left(\zeta_{v} b\right)+\delta d b .
$$

Proof. In (14.6), we put $F=0$ and use the relation $w^{i}{ }_{j} w^{j}=0, w^{i}{ }_{i}=0$. Then Proposition 14.2 follows. 
COROLlARY 14.3. Besides the assumptions on $\zeta_{U}$ as in Proposition 14.2, we suppose that $M$ is of constant scalar curvature and $u$ is an infinitesimal $(m-1)^{s}$-homothety such that $b$ is also constant. Then we have

$$
a R+b R_{1}\left(\zeta_{U}, \zeta_{U}\right)=0 .
$$

Particularly,

(1) If $M$ is an Einstein space, we have $(a m+b) R=0$. So if $R \neq 0$, we get $a m+b=0$.

(2) If ${ }^{\varepsilon} \zeta$ is a parallel field and $R \neq 0$, then $u$ is an infinitesimal $(m-1)^{s}$ isometry.

Propositions 14.1 and 14.2 are useful in $\$ 16$.

The properties of an infinitesimal $(\mathrm{m}-1)$-conformal transformation, which leaves $R, R_{j k}$, or $R_{j k l}^{i}$ invariant respectively, will be studied in other papers.

15. Lie algebras of infinitesimal $(m-1)$-conformal transformations and Lie transformation groups. In this section, we prove that the groups of certain [m-1]-conformal transformations are Lie groups, if the Riemannian manifold satisfies some conditions.

Let $u$ be an infinitesimal [ $m-1]$-conformal transformation:

$$
\begin{aligned}
& L(u) g=a g+w \otimes F+F \otimes w+b w \otimes w, \\
& L(u) w=c w .
\end{aligned}
$$

Then we have a local 1-parameter group $\boldsymbol{\varphi}_{l}(|t|<q(x))$ of local transformations of $M$ :

$$
u_{x}=\lim _{t \rightarrow 0} \frac{\boldsymbol{\varphi}_{t} x-x}{t}
$$

where $q$ is a positive function on $M$. We fix a point $x_{0}$, a positive number $q_{0}$ and neighborhoods $U$ and $V$ of $x_{0}$ satisfying $\boldsymbol{\phi}_{t} V \subset U$, for any $t:|t|<q_{0}$ $<q\left(x_{0}\right)$. As a first step, we consider maps $\varphi_{t}: V \rightarrow \phi_{t} V$.

LEMMA 15.1. There exists a family of differentiable functions $\gamma_{\iota}(|t|$ $<q_{0}$ ) on $V$ such that $\phi_{t}{ }^{*} w=\gamma_{t} w$.

Proof is standard and similarly done as the proof of Lemma 15.2, so we shall omit it.

LEMMA 15.2. Each $\boldsymbol{\varphi}_{t}\left(|t|<q_{0}\right)$ is an $(m-1)$-conformal transformation of $V$ onto $\boldsymbol{\varphi}_{t} V$. 
Proof. First let $X, Y, A, B$ be any tangent vectors at $x_{0}$ which belong to the distribution $D_{x_{0}}$, such that the inner products of $X, Y$ and $A, B$ are not zero. Then we have a real number $\lambda$ such that $g_{x_{0}}(X, Y)=\lambda g_{x_{0}}(A, B)$. We prove $g_{\varphi_{t} x_{0}}\left(\boldsymbol{\varphi}_{t} X, \boldsymbol{\varphi}_{t} Y\right)=\lambda g_{\varphi_{t} x_{0}}\left(\boldsymbol{\varphi}_{t} A, \boldsymbol{\varphi}_{t} B\right)$, for this purpose we put

$$
\Xi(t)=\left(\boldsymbol{\varphi}_{t}^{*} g\right)(X, Y)-\lambda\left(\boldsymbol{\varphi}_{t}^{*} g\right)(A, B) .
$$

It is clear by definition that $\Xi(0)=0$. As $\Xi$ is a function of $t\left(\Xi:\left(-q_{0}, q_{0}\right)\right.$ $\rightarrow R$ ), we can differentiate it and get

$$
\begin{aligned}
& \frac{d \Xi}{d t}=\lim _{s \rightarrow 0} \frac{\boldsymbol{\phi}_{t+s}{ }^{*} g-\boldsymbol{\phi}_{t}^{*} g}{s}(X, Y)-\lambda \lim _{s \rightarrow 0} \frac{\boldsymbol{\phi}_{t+s}{ }^{*} g-\boldsymbol{\phi}_{t}^{*} g}{s}(A, B) \\
& =\lim _{\boldsymbol{s} \rightarrow 0} \frac{\boldsymbol{\varphi}_{s}{ }^{*} g-g}{\boldsymbol{s}}\left(\boldsymbol{\phi}_{t} X, \boldsymbol{\varphi}_{t} Y\right)-\lambda \lim _{\boldsymbol{s} \rightarrow 0} \frac{\boldsymbol{\phi}_{s}{ }^{*} g-g}{\boldsymbol{s}}\left(\boldsymbol{\varphi}_{t} A, \boldsymbol{\varphi}_{t} B\right) \\
& =(L(u) g)\left(\boldsymbol{\varphi}_{t} X, \boldsymbol{\varphi}_{t} Y\right)-\lambda(L(u) g)\left(\boldsymbol{\varphi}_{t} A, \boldsymbol{\varphi}_{t} B\right) \\
& =\operatorname{ag}\left(\boldsymbol{\phi}_{t} X, \boldsymbol{\varphi}_{t} Y\right)-\lambda a g\left(\boldsymbol{\phi}_{t} A, \boldsymbol{\varphi}_{t} B\right),
\end{aligned}
$$

since $\boldsymbol{\phi}_{t} X, \boldsymbol{\varphi}_{t} Y, \boldsymbol{\varphi}_{t} A$ and $\boldsymbol{\varphi}_{t} B$ belong to $D_{\varphi x_{0}}$ by Lemma 15.1. Therefore we get

$$
\frac{d \Xi}{d t}=a\left(\phi_{t} x_{0}\right) \Xi(t) .
$$

This means that $\Xi$ is of the form $p e^{\text {fadt }}, p$ denoting a constant. By $\Xi(0)=0$, we have $\Xi(t)=0$ identically. Thus we get

$$
\frac{\left(\boldsymbol{\phi}_{t}^{*} g\right)(X, Y)}{g(X, Y)}=\frac{\left(\boldsymbol{\phi}_{t}^{*} g\right)(A, B)}{g(A, B)}
$$

for all $X, Y, A, B \in D_{x_{0}}, g(X, Y) \neq 0, g(A, B) \neq 0$. And $\phi_{t}$ is an (m-1)-conformal transformation.

LEMMA 15.3. If $u$ is an infinitesimal $[m-1]^{s}$-conformal transformation, then $\varphi_{t}$ is an $(m-1)^{s}$-conformal transformation of $V_{t}$ onto $\varphi_{t} V$.

PROOF. By Lemma 15.2, we have $\alpha_{t}, \beta_{t}$ and $\theta_{t}$ of functions and 1 -forms on $V$ such that

$$
\boldsymbol{\varphi}_{t}^{*} g=\alpha_{t} g+w \otimes \theta_{t}+\theta_{t} \otimes w+\beta_{t} w \otimes w .
$$

We prove $\theta_{t}=0$. Let $X$ be any tangent vector $x_{0}$ belonging to $D_{x_{0}}$. Then 
$t \rightarrow \theta_{t}(X)$ defines a function $\Xi^{\prime}:\left(-q_{0}, q_{0}\right) \rightarrow R$. As $\theta_{t}(X)=\left(\varphi_{t}^{*} g\right)(\zeta, X)$, we have

$$
\begin{aligned}
\frac{d \Xi^{\prime}}{d t} & =\lim _{s \rightarrow 0} \frac{\boldsymbol{\varphi}_{s}^{*} g-g}{s}\left(\boldsymbol{\phi}_{t} \zeta, \boldsymbol{\varphi}_{t} X\right) \\
& =a\left(\boldsymbol{\phi}_{t} x_{0}\right) \Xi^{\prime}(t) .
\end{aligned}
$$

Thereby $\Xi^{\prime}(t)=0$ holds and so $\theta_{t}=0$ follows.

LEMMA 15.4. If $u$ is an infinitesimal [m-1]-homothety, then $\phi_{t}$ is an (m-1)-homothety. In particular, if $u$ is an infinitesimal [m-1]-isometry, then $\varphi_{l}$ is an $(m-1)$-isometry.

ProOF. We put

$$
\Xi^{\prime \prime}(t, x)=\alpha_{t}(x) \quad|t|<q_{0}, x \in M .
$$

Then we have

$$
\frac{\partial \Xi^{\prime \prime}}{\partial t}(t, x)=\lim _{s \rightarrow 0} \frac{\alpha_{s}\left(\phi_{t} x\right) \alpha_{t}(x)-\alpha_{t}(x)}{s},
$$

since $\alpha_{t+s}(x)=\alpha_{s}\left(\phi_{t} x\right) \alpha_{t}(x)$ by (9.4). Thus we get

$$
\begin{aligned}
\frac{\partial \Xi^{\prime \prime}}{\partial t}(t, x) & =\alpha_{t}(x) \frac{\partial \Xi^{\prime \prime}}{\partial t}\left(0, \phi_{t} x\right) \\
& =a \Xi^{\prime \prime}(t, x)
\end{aligned}
$$

because by assumption, $\frac{\partial \Xi^{\prime \prime}}{\partial t}(0, x)=a=$ constant. And as a solution of (15.5), we have

$$
\Xi^{\prime \prime}(t, x)=f(x) e^{a t},
$$

where $f$ is a function on $V$ independent of $t$. On the other hand $\Xi^{\prime \prime}(0, x)$ $=\alpha_{0}(x)=1$, and so $f(x)=1$. This shows that $\Xi^{\prime \prime}(t, x)=\Xi^{\prime \prime}(t)$ is constant $e^{a t}$ on $V$ for each $t:|t|>q_{0}$. In particular, if $a=0$, then $\alpha_{t}=1$.

Similarly we can prove

LEMMA 15.5. If $c$ is constant, then $\gamma_{t}$ in Lemma 15.1 is constant. 
We use the notations:

$$
\begin{aligned}
& \mathfrak{P}=\{u: \text { infinitesimal }[m-1] \text {-conformal transformation }\}, \\
& \mathfrak{S}=\{u: \text { infinitesimal }[m-1] \text {-homothety }\} \\
& \mathfrak{S}=\{u: \text { infinitesimal }[m-1] \text {-isometry }\}, \\
& \mathfrak{P}^{s}=\left\{u: \text { infinitesimal }[m-1]^{s} \text {-conformal transformation }\right\} .
\end{aligned}
$$

And we put

$$
\mathfrak{S}^{s}=\mathfrak{g}_{\mathfrak{2}} \cap \mathfrak{P}^{s}, \quad \mathfrak{J}^{s}=\mathfrak{\Im} \cap \mathfrak{P}^{s}
$$

By definition we have $\mathfrak{P} \supset \mathfrak{S} \supset \mathfrak{J}$, concerning a bracket operation, we have

PROPOSITION 15.6.

$$
[\mathfrak{P}, \mathfrak{P}] \subset \mathfrak{B}, \quad\left[\mathfrak{P}^{s}, \mathfrak{P}^{s}\right] \subset \mathfrak{P}^{s} .
$$$$
[\mathfrak{S}, \mathfrak{S}] \subset \mathfrak{J}, \quad\left[\mathfrak{G}^{s}, \mathfrak{S}^{s}\right] \subset \mathfrak{S}^{s} .
$$

$[\mathfrak{\Im}, \mathfrak{\Im}] \subset \mathfrak{J}, \quad\left[\mathfrak{\Im}^{s}, \mathfrak{\Im}^{s}\right] \subset \mathfrak{\Im}^{s}$.

By preceding Lemmas, we have

Proposition 15.7. If $u$ is an element of $\mathfrak{B}, \mathfrak{S}, \mathfrak{J}, \mathfrak{P}^{s}, \mathfrak{f}^{s}$ or $\mathfrak{J}^{s}$ and generates a 1-parameter group $\phi_{t}(t \in R)$ of global transformations of $M$, then each $\phi_{t}$ belongs to $\Pi, \Theta, \Phi, \Pi^{s}, \Theta^{s}$ or $\Phi^{s}$ respectively.

LEMMA 15.8. Let $u$ be an infinitesimal transformation such that $L(u) g$ $=a g+b w \otimes w$, where $b$ is a constant. Then the set of all such $u$ is finite demensional.

ProOF. Let $u$ be an element of the set such that $b$ is not zero. We define $\bar{u}$ by $\bar{u}=(1 / b) u$, then

$$
L(\bar{u}) g=a_{0} g+w \otimes w
$$

where $a_{0}$ is a differentiable function on $M$. Then for any element $v$ of the set :

$$
L(v) g=a^{\prime} g+b^{\prime} w \otimes w,
$$

we have

$$
v=\left(v-b^{\prime} \bar{u}\right)+b^{\prime} \bar{u}
$$


where $v-b^{\prime} \bar{u}$ is an infinitesimal conformal transformation. Thus the set of such $v-b^{\prime} \bar{u}$ is finite dimensional, whence the set of such $v$ is also finite demensional.

THEOREM 15.9. The subgroup of $\Theta^{s}$, whose element satisfies $\phi^{*} w_{V}=\gamma_{V U} w_{U}$ for some constant $\gamma_{v U}$, is a Lie group.

Proof. By R. S. Palais' theorem [13], it is enough to prove finite dimensionality of the Lie subalgebra of $\tilde{F}^{s}$, whose element generates a 1 parameter group of global $[m-1]^{s}$-homotheties which satisfy $\boldsymbol{\phi}_{t}{ }^{*} w_{V}=\gamma_{t V U} w_{U}$ for some constant $\gamma_{t V U}$ for each $t \in R$.

Any element $u$ of the Lie subalgebra satisfies $L(u) w=c w$ and $L(u) g=a g$ $+b w \otimes w$, where $c$ and $a$ are constant. Then $b$ is also constant. Thus by Lemma 15.8, the Lie subalgebra is finite dimensional.

LEMMA 15.10. If $\zeta_{U}$ is a Killing vector field for each $U$ and if $u$ is an element of $\mathfrak{B}^{s}$. Then $L\left(\zeta_{U}\right) a=0$.

PROOF. Taking the Lie derivative of $L(u) g$ with respect to $\zeta$ we have

$$
L(\zeta) L(u) g=\zeta a \cdot g+\zeta b \cdot w \otimes w
$$

where we have used $L(\zeta) g=0$ and $L(\zeta) w=0$. And as

$$
L(c \zeta)=-L([u, \zeta])=-L(u) L(\zeta)+L(\zeta) L(u)
$$

and $L(c \xi) g=d c \otimes w+w \otimes d c$, we have

$$
d c \otimes w+w \otimes d c=\zeta a \cdot g+\zeta b \cdot w \otimes w
$$

In the above equation each term excepting $\zeta a \cdot g$ contains $w$, so we see that $\zeta a=0$.

LEMMA 15.11. Suppose that the distribution defined by ${ }^{\text {g }} \mathrm{g}$ is regular, $\zeta_{U}$ is a Killing vector field, and each trajectory of ${ }^{\varepsilon} \zeta$ is complete. Then the set $M /{ }^{\varepsilon} \zeta=\tilde{M}$ of all trajectories of ${ }^{\varepsilon} \zeta$ becomes a Riemannian manifold and each $u \in \mathfrak{P}^{s}$ induces an infinitesimal conformal transformation $\widetilde{u}$ on $\tilde{M}$.

Proof. Following [21], first we assume that there exists a point $x$ of $M$ such that the trajectory $l(x)$ which passes through $x$ is closed. Then we have the length $s=|l(x)|$ of $l(x)$, and we take a sufficiently small tubular neighborhood $W=W(l(x))$ of $l(x)$. 
On $W$ we can define a vector field $\bar{\zeta}$ such that $\left.\bar{\zeta}\right|_{U}=\zeta_{I}$ or $-\zeta_{U}$ for each $U$ if $U \cap W$ is non-empty. Then $\bar{\zeta}$ is a Killing vector field on $W$ and generates a 1-parameter group $\phi_{t}(t \in R)$ of isometries of $W$. And as $\bar{\zeta}$ is also regular, we can conclude that $\phi_{s}$ is an identity transformation of $W$ and each trajectory of $\bar{\zeta}$ and hence $\xi$ is of constant length $s$ ([21]).

Therefore either all trajectories of $\xi \xi$ are homeomorphic to a circle, or all trajectories are homeomorphic to the real line $R$. By [13] or [21], $M /{ }^{\circ} \xi$ is a differentiable manifold which has Riemannian metric $h$ such that $g=\pi^{*} h$ $+w \otimes w, \pi$ denoting the natural projection: $M \rightarrow M / \varepsilon \xi=\widetilde{M}$.

It may be remarked that if $\varepsilon \xi$ is a globally defined vector field, then $M / \varepsilon \xi$ is a principal fiber bundle.

Now let $u \in \mathfrak{P}^{s}$. As $L(\zeta) u=-L(u) \xi=c \zeta$, by the differential $\pi$ of $\pi, \pi u=\widetilde{u}$ is a vector field on $\tilde{M}$. Denote by $\varphi_{t}$ and $\widetilde{\varphi}_{t}$ the (local) 1-parameter groups of (local) transformations generated by $u$ and $\widetilde{u}$, then they satisfy $\pi \varphi_{t}=\widetilde{\varphi}_{t} \pi$.

Using the fact that $\phi_{t}$ is an $[m-1]^{s}$-conformal transformation, we have

$$
\begin{aligned}
\left(\widetilde{\varphi}_{t}^{*} h\right)(X, Y) & =h\left(\widetilde{\varphi}_{t} X, \widetilde{\varphi}_{t} Y\right) \\
& =h\left(\pi \varphi_{t} \pi^{-1} X, \pi \varphi_{t} \pi^{-1} Y\right) \\
& =\left(\phi_{t}^{*}\left(\pi^{*} h\right)\right)\left(\pi^{-1} X, \pi^{-1} Y\right)
\end{aligned}
$$

for any tangent vectors $X, Y$ at $\tilde{x} \in \tilde{M}$, where we consider $\pi^{-1} X$ as a tangent vector at $x \in \widetilde{x}$ such that $w\left(\pi^{-1} X\right)=0$ and $\pi\left(\pi^{-1} X\right)=X$. Of course $\pi^{-1} X$ at $x$ is uniquely determined and we can prove that the value of $h_{\tilde{\varphi}_{t} \tilde{x}}\left(\pi_{\varphi x} \varphi_{t x} \pi^{-1} X_{\tilde{x}}\right.$, $\left.\pi_{\varphi x} \boldsymbol{\varphi}_{t x} \pi^{-1} Y_{\tilde{x}}\right)$ does not depend on the choice of $x \in \tilde{x}$ and the choice of $\pi^{-1} X$ or $\pi^{-1} Y$ so far as $\pi\left(\pi^{-1} X\right)=X$ and $\pi\left(\pi^{-1} Y\right)=Y$ are satisfied, because the difference is of the form $k \zeta$, for some real number $k$. Then, as

$$
\begin{aligned}
\boldsymbol{\varphi}_{t}^{*}\left(\pi^{*} h\right) & =\boldsymbol{\varphi}_{t}^{*}(g-w \otimes w) \\
& =\alpha_{t} g+\beta_{t} w \otimes w-\gamma_{t}^{2} w \otimes w,
\end{aligned}
$$

we obtain

$$
\begin{aligned}
\left(\widetilde{\varphi}_{t}^{*} h\right)(X, Y) & =\left(\alpha_{t} g\right)\left(\pi^{-1} X, \pi^{-1} Y\right) \\
& =\left(\alpha_{t} \pi^{*} h+\alpha_{t} w \otimes w\right)\left(\pi^{-1} X, \pi^{-1} Y\right) \\
& =\alpha_{t} h(X, Y) .
\end{aligned}
$$

Notice here that $\alpha_{t}$ is constant on each trajectory of ${ }^{\varepsilon} \xi$. Namely by Lemma 15.10, we have $\zeta a=0$ and by the almost similar method in Lemma 15.4, we 
can show that $\zeta \alpha_{t}=0$. Therefore $\widetilde{u}$ is an infinitesimal conformal transformation on $\tilde{M}$.

THEOREM 15.12. Suppose that the 1-dimensional distribution by ${ }^{\mathrm{E}} \boldsymbol{\xi}$ is regular, $\zeta_{U}$ is a Killing vector field and each trajectory of ${ }^{\xi} \zeta$ is complete. Then the subgroup $\Pi^{\text {sc }}$ of $\Pi^{\text {s }}$ which consists of the element $\phi$ satisfying $\phi^{*} w_{V}=\gamma_{V U} w_{U}$ on $U \cap \phi^{-1} V$ for some constant $\gamma_{V U}$ is a Lie group.

PROOF. We devide the proof into two parts.

$\left(1^{\circ}\right)$ The case: ${ }^{\circledR} \zeta$ is not a parallel field.

Denote by $\mathfrak{P}^{s c}$ the Lie subalgebra of $\mathfrak{P}^{s}$ which consists of all $u$ satisfying $L(u) w=c w$ for some constant $c$. The map $\pi: u \rightarrow \widetilde{u}$ gives a homomorphism of $\mathfrak{P}^{s}$, also of $\mathfrak{P}^{s c}$, into the set of all infinitesimal conformal transformations on $\tilde{M}$ as Lie algebras. The kernel of $\pi$ is the set of the form ${ }^{\varepsilon} f^{\varepsilon} \xi$ for some ${ }^{\varepsilon} f, f_{r}$ is a scalar field on each $U$. As $\zeta_{U}$ is a Killing vector field, ${ }^{\varepsilon} f^{\varepsilon} \xi$ belongs to $\mathfrak{A}^{s c}$ if and only if $L(f \zeta) w=d f=r w$ for some constant $r$. Taking the exterior differentiation of $d f_{U}=r w_{U}$, we have $r d w_{U}=0$ on each $U$. However, ¿ $\zeta$ is a Killing vector field and not a parallel field, there exists $U$ on which $d w_{0} \neq 0$. Consequently we get $r=0$. Then we have $d f_{U}=0$ for each $U$. So $\left|{ }^{\varepsilon} f\right|$ of ${ }^{\varepsilon} f^{\varepsilon} \zeta$ is constant. Of course as ${ }^{\varepsilon} f^{\varepsilon} \zeta$ must be a vector field, if $M$ admits non-trivial ${ }^{\varepsilon} f$ we can assume that $\zeta$ is a globally defined vector field by suitable choice of $\zeta_{V},-\zeta_{V}$. And so the kernel is given by $\{t \zeta, t \in R\}$. Thus, as the set of all infinitesimal conformal transformations on $\tilde{M}$ is finite dimensional, $\mathfrak{P}^{s c}$ is also finite dimensional.

$\left(2^{\circ}\right)$ The case: ${ }^{\varepsilon} \zeta$ is a parallel field.

In this case, we take the universal covering manifold $\bar{M}$ of $M$ and define $\bar{g}, \varepsilon \bar{\zeta}, \varepsilon_{\bar{w}}$ and $\bar{u}$ for $u \in \mathfrak{P}^{s c}$ naturally on $\bar{M}$ by the local diffeomorphisms. Then $\bar{u}$ is also an infinitesimal $[m-1]^{s}$-conformal transformation on $\bar{M}$. So it suffices to show the finite dimensionality of the set $\{\bar{u}\}$ in $\bar{M}$. As ${ }^{\varepsilon} \bar{\zeta}$ is parallel and $\bar{M}$ is simply connected we may assume that $\bar{\zeta}$ is a globally defined vector field on $\bar{M}$. And it is easy to see that $\bar{\zeta}$ is also regular, so we have $\bar{M} / \bar{\zeta}$ and a Riemannian metric $\bar{h}$ on $\bar{M} / \bar{\zeta}$. $\bar{M} / \bar{\zeta}$ is also simply connected, and $\bar{M}$ is a principal fiber bundle over $\bar{M} / \bar{\zeta}$. $\bar{\omega}$ defines an infinitesimal connection on $\bar{M}$, and $w=0$ is completely integrable. Similarly to the case $\left(1^{\circ}\right)$, we consider the projection of $\bar{u}$ by the projection $\bar{M} \rightarrow \bar{M} / \bar{\zeta}$, and study its kernel. Then any element of the kernel is of the form $\bar{f} \bar{\zeta}$ for some scalar field $\bar{f}$ satisfying $d \bar{f}=r \bar{w}$ for some constant $r$. As a special case we take $r=1$, then the solution $\bar{f}_{0}$ of $\bar{w}=d \bar{f}$ is uniquely determined, if we fix a horizontal global section $S$ in $\bar{M}$ and give the initial condition $\bar{f}_{0}=1$ on $S$, because $\bar{f}$ is constant on each horizontal section. So general solution of $d \bar{f}=r \bar{w}$ is $\bar{f}=r \overline{f_{0}}+s$ for constant $r$ and $s$. That is to say the kernel is $\left\{r\left(\bar{f}_{0} \bar{\zeta}\right)+s \bar{\zeta}: r, s \in R\right\}$ and 
at most 2-dimensional, Thus $\mathfrak{B}^{s c}$ is finite dimensional. Therefore in both cases $\left(1^{\circ}\right),\left(2^{\circ}\right), \Pi^{s c}$ makes a Lie group.

REMARK 1. If $M$ admits an element $u$ in $\mathfrak{P}^{s c}$ such that $L(u) w=c w$ for a constant $c \neq 0$. Then ${ }^{\varepsilon} \zeta$ is necessarily regular (see [18], §4).

PEMARK 2. In $\left(1^{\circ}\right)$ above, if $\boldsymbol{\xi} \zeta$ cannot define a globally difined vector field by any choice of $\zeta_{U},-\zeta_{V}$. Then the dimension of $\mathfrak{P}^{s c}$ is not greater than that of the set of all infinitesimal conformal transformations on $\tilde{M}$.

PEMARK 3. In the above Theorem, if $M$ is complete, then each trajectory of ${ }^{\varepsilon} \zeta$ is complete.

16. The cases where $M$ is compact and the scalar curvature $R$ is constant. In the first place, we prove general theorems.

THEOREM 16.1. Suppose that $M$ is compact and orientable and $u$ is an infinitesimal $(m-1)$-conformal transformation, then we have

$$
\int_{M}(a m+b) d \sigma=0 \text {. }
$$

PROOF. Contracting (13.1) with $g^{i j}$ and noticing that $L(u) g_{i j}=u_{i, j}+u_{j, i}$, we get

$$
2 u^{i}{ }_{i}=a m+b .
$$

Integration of the last equation over $M$ is (16.1).

In the following, we denote the left hand side of (16.1) by a global inner product $\langle a m+b, 1\rangle$.

Definition. We call $M$ a $\zeta$-space, if

(i ) $\delta w_{U}=0$, (i.e. $\xi_{U}$ : volume-preserving),

(ii) $\nabla_{\xi_{U}} \zeta_{U}=0$, (i.e. each trajectory of $\zeta_{U}$ is a geodesic),

(iii) $R_{1}\left(\zeta_{U}, \zeta_{U}\right)=T=$ constant

for each $U$. If $M$ satisfies only (i) and (ii), then we say that $M$ has properties (i) and (ii).

EXAmPLES. $\left(1^{\circ}\right) K$-contact manifold is a $\zeta$-space such that $T=\frac{m-1}{4}$ ([17], p. 329). 
$\left(2^{\circ}\right)$ If a manifold $M$ admits a parallel direction field ${ }^{\varepsilon} \xi$. Then $M$ is a $\zeta$-space with $T=0$.

THEOREM 16.2. Suppose that $M$ is compact and orientable and has properties (i) and (ii), if $L(u) w_{U}=c w_{U}$, then

$$
<c, 1>=0 .
$$

Further if $u$ is an infinitesimal $[m-1]^{s}$-conformal transformation then re have

$$
<a, 1>=0, \quad<b, 1>=0 .
$$

PROOF. Expression of $L(u) w=c w$ by local coordinates is as follows:

$$
w_{i, r} u^{r}+w_{r} u_{, i}^{r}=c w_{i} .
$$

Transvecting (16.4) with $w^{i}$, we have

$$
c=w_{r} u_{, i}^{r} w^{i}=\left(w_{r} u^{r} w^{i}\right)_{, i}
$$

because $w^{i}{ }_{i}=0$ and $w_{r, i} w^{i}=0$. Although $w$ is not a globally defined tensor, $\left(w_{r} u^{r} w^{i}\right)$ is a globally defined vector field. So if we integrate (16.5) over $M$, we have (16. 2). By Theorem 12.1, if $u$ is an $[m-1]^{s}$-conformal transformation, we have $2 c=a+b$. Thus

$$
<a+b, 1>=0 .
$$

Then (16.1) and (16.6) yield (16.3).

COROLLARY 16.3. In a compact orientable $M$ with properties (i) and (ii), if the scalar field $c$ in $L(u) w_{U}=c w_{U}$ is constant, then $c=0$.

THEOREM 16.4. In a compact orientable $M$ with properties (i) and (ii), every infinitesimal $[m-1]^{s}$-homothety is an infinitesimal $[m-1]^{s}$-isometry.

Proof. This is an immediate consequence of Theorem 16.2.

LEMMA 16.5. If $M$ is compact and orientable, and if the scalar curvature $R$ is constant, we have

$$
<a R+b T, 1>=0
$$


for any infinitesimal $(m-1)^{s}$-conformal transformation $u$.

PROOF. (16.7) follows from Proposition 14.1.

LEMMA 16.6. In a compact orientable $M$ with properties (i) and (ii), we have

$$
\begin{aligned}
(m-1)<d a, d a>-<a, a R+b T & >-<a, L(u) R> \\
& +<d a-(\xi a) w, d b-(\xi b) w>=0
\end{aligned}
$$

for any infinitesimal $(m-1)^{s}$-conformal transformation $u$.

ProOF. As $\delta$ is dual to $d$, we have

$$
<d a, d a>-<a, \delta d a>=0
$$

On the other hand, by virtue of Proposition 14.2, we get

$$
(m-1) \delta d a=a R+b T+L(u) R-\delta d b-\zeta(\zeta b)
$$

And we get

$$
\begin{aligned}
<a, \delta d b> & =\langle d a, d b> \\
<a, \xi(\xi b)> & =\langle a w, d(\zeta b)> \\
& =-\langle\xi a, \zeta b>
\end{aligned}
$$

since $\delta(a w)=a \delta w-\zeta a$ and $\delta w=0 . \quad$ Moreover

$$
\langle d a-(\zeta a) w, d b-(\zeta b) w>=\langle d a, d b>-<\zeta a, \zeta b>.
$$

Substitution $\delta d a$ of (16.10) into (16.9) using above relations yields (16.8).

LEMMA 16.7. In a compact orientable $M$, if $w_{0}$ is a closed form, then we have

$$
\langle d a-(\zeta a) w, d b-(\xi b) w>=-<d a, d a>+\langle\zeta a, \zeta a\rangle
$$

for any infinitesimal $[m-1]^{s}$-conformal transformation.

PROOF. (16.12) is valid always with respect to the (local) inner product which we denote by $($,$) . So we prove here (16.12) for the inner product.$ 
As $L(u) w=c w$ for some scalar field $c$, we have $d L(u) w=d c \wedge w+c d w$ by exterior differentiation, where $\wedge$ denotes the exterior product. Since $d$ and $L(u)$ are commutative and $d w=0, d c \wedge w=0$ follows. Thus $d c$ is proportional to $w$ and $d c=\zeta c \cdot w$. By Theorem 12.1, we have $d a+d b=\zeta(a+b) \cdot w$. And so we consider the inner product with $d a$, and get

$$
(d a, d a+d b)=(\zeta a, \zeta a+\zeta b),
$$

from which we have

$$
(d a, d b)-(\zeta a, \zeta b)=-(d a, d a)+(\zeta a, \zeta a)
$$

Here we notice that (16.11) holds also with respect to the inner product. Then, from (16.11) and (16.13), relation (16.12) for the inner product follows.

LEMMA 16.8. As for $T$ we have;

If $w_{U}$ is a harmonic form,

$$
T=-2(\nabla w, \nabla w) \leqq 0 .
$$

If $\zeta_{U}$ is a Killing vector field,

$$
T=2(\nabla w, \nabla w) \geqq 0 .
$$

Proof is easy, since $\zeta_{U}$ is a unit vector field.

As a general statement, we have

PROPOSITION 16.9. In a compact orientable $M$, we assume that w is a harmonic form for each $U$. Then an infinitesimal $[m-1]^{s}$-conformal transformation $u$ is an infinitesimal $[m-1]^{s}$-isometry if and only if it satisfies

$$
<a, a R+b T+L(u) R>\leqq 0
$$

PROOF. If $w$ is a harmonic form, we have $d w=0$ and $\delta w=0$. The length of $w$ being equal to $1, \nabla_{\xi} \zeta=0$ follows from $d w=0$. Then, by Lemma 16.6 and 16.7 , we have

$$
(m-2)<d a, d a>-<a, a R+b T+L(u) R>+\langle\xi a, \zeta a>=0
$$

If (16.16) holds, (16.17) means that each term is zero. So $d a=0$ follows, that is $a$ is constant. Moreover, by (16.3) ${ }_{1}$ in Theorem 16.2, $a$ is equal to zero. q.e.d. 
If $\zeta$ is a parallel field, $M$ is a $\zeta$-space with $T=0$. Therefore we get

THEOREM 16.10. In a compact orientable $M$, if $\varepsilon \xi$ is a parallel field and $R=$ constant $\leqq 0$. Then any infinitesimal $[m-1]^{s}$-conformal transformation is an infinitesimal $[m-1]^{\text {s-isometry. }}$

REMARK 1. In the above Theorem, essencially we need the condition that a compact orientable $M$ is a $\zeta$-space satisfying $T=0$, $d w=0$ and $R=$ constant $\leqq 0$. However, if $\zeta$-space satisfies $d w=0, w_{U}$ is a harmonic form. So $T$ is non-positive by Lemma 16.8. Thus if $T=0, w_{U}$ is necessarily a parallel field.

Next we consider the case where an infinitesimal $[m-1]^{s}$-conformal transformation $u$ satisfies $c=0$. Of course, the only possible case of $c=$ constant is the case $c=0$ in the manifold with properties (i) and (ii) by Theorem 16.2.

Now as $2 c=a+b$, we have $d a=-d b$. On the other hand

$$
<d a-(\zeta a) w, d a-(\zeta a) w>\leqq<d a, d a>\text {. }
$$

If we utilize (16.8) and (16.18), we get

$$
(m-2)<d a, d a>-<a, a R-a T+L(u) R>\leqq 0
$$

So, if the second term is non-negative, we have $d a=0$ and $a=0$. Consequently we have also $b=0$ and $u$ is a Killing vector field. Thus we have

PROPOSITION 16.11. In a compact orientable $M$ with properties (i) and (ii), an infinitesimal $[m-1]^{s}$-conformal transformation $u$ such that $L(u) w=0$ is an infinitesimal isometry if and only if it satisfies

$$
<a, a R-a T+L(u) R>\leqq 0 .
$$

THEOREM 16.12. In a compact orientable $M$, if $\zeta_{U}$ is a Killing vector field and $R=$ constant $\leqq 0$. Then any infinitesimal $[m-1]^{s}$-conformal transformation $u$ satisfying $L(u) w=c w$ for some constant $c$ is a Killing vector filed.

PROOF. As $\zeta$ is a unit and Killing vector field, $M$ has properties (i) and (ii). $\quad c=0$ follows from Corollary 16.3. By (16.15) in Lemma 16.8, $T$ is nonnegative. And $R$ is a non-positive constant, (16.20) holds good. Then by Proposition 16.11, $u$ is a Killing vector field. 


\section{BIBLIOGR APHY}

[1] S. IsHIHARA, Groups of projective transformations and groups of conformal transformations, Journ. Math. Soc. Japan, 9(1957), 195-227.

[2] S. ISHIHARA AND M. OBATA, Affine transformations in a Riemannian manifold, Tôhoku Math. Journ., 7(1955), 146-150.

[3] M. S. KNEBelman And K. Yano, On homothetic mappings of Riemann spaces, Proc. Amer. Math. Soc., 12(1961), 300-303.

[4] S. KoBAYASHI. Le groupe des transformations qui laissent invariant le paraléllisme, Colloque de Top. de Strasbourg, (1954).

[5] - A theorem on the affine transformation group of a Riemannian manifold, Nagoya Math. Journ., 9(1955), 39-41.

[6] S. KOBAYASHI AND K. NomizU, Foundations of differential geometry, Interscience Pub. New York, 1963.

[7] A. LiChNEROWICZ, Géométrie des groupes de transformations, Dunod Paris, 1958.

[8] H. Mizusawa, On infinitesimal transformations of $K$-contact and normal contact metric spaces, Sci. Rep. Niigata Univ. Ser. A, 1(1964), 5-18.

[9] On certain infinitesimal conformal transformations of contact metric spaces, Sci. Rep. Niigata Univ., Ser. A, 2(1965), 33-39.

[10] M. OBATA, Conformal transformations of compact Riemannian manifolds, Illinois Journ. Math., 6(1962), 292-295.

[11] M. OKumURA, Some remarks on spaces with a certain contact structure, Tôhoku Math. Journ., 14(1962), 135-145.

[12] On infinitesimal conformal and projective transformation of normal contact spaces, Tôhoku Math. Journ., 14(1962), 398-412.

[13] R. S. PALAIS, A global formulation of the Lie theory of transformation groups, Memoirs of Amer. Math. Soc., 22(1957).

[14] S. SASAKI, On differentiable manifolds with certain structures which are closely related to almost contact structure, I, Tôhoku Math. Journ., 12(1960), 459-476.

[15] S. SASAKI AND Y. HATAKEYAMA, On differentiable manifolds with certain structures which are closely related to almost contact structure, II, Tôhoku Math. Journ., 13 (1961), 281-294.

[16] T. Sumitomo, Projective and conformal transformations in compact Riemannian manifolds, Tensor (N. S. ), 9(1959), 113-135.

[17] S. TANNO, Some transformations on manifolds with almost contact and contact metric structures, I, II, Tôhoku Math. Journ., 15(1963), 140-147, 322-331.

[18] - On fiberings of some non-compact contact manifolds, Tôhoku Math. Journ., 15(1963), 289-297.

[19] — A remark on transformations of a $K$-contact manifolds, Tôhoku Math. Journ., 16(1964), 173-175.

[20] Sur une variété munie d'une structure de contact admettant certaines transformations, Tôhoku Math. Journ., 17(1965), 239-243.

[21] - A theorem on regular vector fields and its applications to almost contact structures, Tôhoku Math. Journ., 17(1965), 235-238.

[22] K. YANO, The theory of Lie derivatives and its applications, Amsterdam, North Holland Pub. Co., 1957.

[23] K. YANo AND S. Bochner, Curvature and Betti Numbers, Annals of Math. Studies 32, Princeton, 1953.

TôHOKU UNIVERSITY. 